\title{
Continuous-time histories: observables, probabilities, phase space structure and the classical limit
}

\author{
Charis Anastopoulos * \\ Department of Physics, University of Maryland, \\ College Park, MD20742, USA
}

October 26, 2018

\begin{abstract}
The continuous-time histories programme stems from the consistent histories approach to quantum theory and aims to provide a fully covariant formalism for quantum mechanics. In this paper we examine some structural points of the formalism. We demonstrate a general construction of history Hilbert spaces and identify a large class of time-averaged observables. We pay particular attention to the construction of the decoherence functional (the object that encodes probability information) in the continuous-time limit and its relation to the temporal structure of the theory. Phase space observables are introduced, through the study of general representations of the history group, which is the analogue of the canonical group in the formalism. We can also define a closed-timepath (CTP) generating functional for each observable, which encodes the information of its correlation functions. The phase space version of the CTP generating functional leads to the implementation of a Wigner-Weyl transforms, that gives a description of quantum theory solely in terms of phase space histories. These results allow the identification of an algorithm for going to the classical (stochastic) limit for a generic quantum system.
\end{abstract}

*charis@physics.umd.edu 


\section{Introduction}

\section{I.1 Canonical vs covariant}

Physical systems can be described in two different ways, depending on one's attitude towards time evolution. The first description can be called "canonical": it focuses on properties of a system at a single moment of time and studies how these properties change. It, therefore, provides an evolutionary picture of physical phenomena. The other type is best described as "covariant": its main objects are histories of the physical system. Its main aim is to find criteria that determine which of them are realizable. As such, this description provides a timeless and (in a sense) teleological picture of physical processes.

In classical mechanics the "canonical" description is Hamilton's formalism. States of the system correspond to points of the phase space, which is a symplectic manifold. Time evolution is implemented by the action of an one-parameter group of symplectic transformations. Alternatively, one can start from the action principle, which provides the covariant description of classical mechanics. Histories are paths, and the physically realized are the ones that minimize the action subject to fixed boundary conditions .

These two approaches also appear in classical probability theory. A physical system at a moment of time is described by a probability distribution on a space $\Omega$ of elementary alternatives. We then study how this distribution evolves in time: the evolution law is a linear partial differential equation, like the Fokker-Planck equation. The "covariant" description of probability theory is provided by the theory of stochastic processes. Here, histories are paths on $\Omega$ and the physical information is encoded in a probability measure $d \mu$ in the space of all histories; it incorporates information about both initial conditions and dynamics.

Quantum theory was developed in the "canonical" framework. The probabilistic information about a system is encoded in a Hilbert space vector, or more generally a density matrix. Its time evolution is given by an one-parameter group of unitary transformations: this is equivalent to Schrödinger's equation. The general structure is very similar to classical probability theory, except for the fact that the observables do not form a commutative algebra.

\section{I.2 Quantum mechanical histories}

When one tries to construct a "covariant" description of quantum theory, a problem immediately arises: the natural probability measure for histories is not additive. This is due to the fact, that quantum theory is based on amplitudes. When one constructs probabilities out these amplitudes, interference between histories appears.

In general, a history corresponds to properties of the physical system at successive instants of time. Since in quantum theory a property (or a proposition about it) is represented by a projection operator, a discrete-time history $\alpha$ will correspond to a string $\hat{\alpha}_{t_{1}}, \hat{\alpha}_{t_{2}}, \ldots \hat{\alpha}_{t_{n}}$ of projectors, each labelled by an instant 
of time. From them, one can construct the class operator

$$
\hat{C}_{\alpha}=\hat{U}^{\dagger}\left(t_{1}\right) \hat{\alpha}_{t_{1}} \hat{U}\left(t_{1}\right) \ldots \hat{U}^{\dagger}\left(t_{n}\right) \hat{\alpha}_{t_{n}} \hat{U}\left(t_{n}\right)
$$

where $\hat{U}(s)=e^{-i \hat{H} s}$ is the time-evolution operator. The probability for the realization of this history is

$$
p(\alpha)=\operatorname{Tr}\left(\hat{C}_{\alpha}^{\dagger} \hat{\rho}_{0} \hat{C}_{\alpha}\right),
$$

where $\hat{\rho}_{0}$ is the density matrix describing the system at time $t=0$.

But this expression does not define a probability measure in the space of all histories, because the Kolmogorov additivity condition cannot be satisfied: if $\alpha$ and $\beta$ are exclusive histories and $\alpha \vee \beta$ denotes their conjunction as propositions, then it is not true that

$$
p(\alpha \vee \beta)=p(\alpha)+p(\beta) .
$$

The histories formulation of quantum theory does not, therefore, enjoy the status of a genuine probability theory.

\section{I.2.1 The consistent histories interpretation}

The formalism sketched above was developed as a part of the consistent histories approach to quantum theory, by Griffiths, Omnés, Gell-Mann and Hartle [1, 2, 3, 4. In this approach, the problem of the non-additivity of the probability measure is addressed by the remark that an additive probability measure is definable, when we restrict to particular sets of histories. These are called consistent sets. They are more conveniently defined through the introduction of a new object: the decoherence functional. This is a complex-valued function of a pair of histories given by

$$
d(\alpha, \beta)=\operatorname{Tr}\left(\hat{C}_{\alpha}^{\dagger} \hat{\rho}_{0} \hat{C}_{\beta}\right) .
$$

A set of exclusive and exhaustive alternatives is called consistent, if for all pairs of different histories $\alpha$ and $\beta$ in the set, we have

$$
d(\alpha, \beta)=0 .
$$

In that case one can use equation (1.2) to assign a probability measure to this set. The consistent histories interpretation then proceeds by postulating that any prediction or retrodiction, we can make based on probabilities has always to make reference to a given consistent set. This leads to counter-intuitive and arguably unphysical situations of getting mutually incompatible predictions, when reasoning within different consistent sets [5, 6]. The predictions of this theory are therefore contextual: but in any case, this is a general feature of all realist interpretations of quantum theory.

Even if the formalism of quantum mechanical histories was originally introduced as part of the consistent histories approach, it is conceptually distinct. 
The same formalism can be viewed in the light of any other interpretational scheme. The Copenhagen interpretation, for instance, would view the nonadditivity of the probability measure in a neutral light. The expression (1.2) describes the statistics of an ensemble of time-ordered sequences of measurements. There would be no a priori theoretical reason for the statistics to correspond to a genuine probability measure.

In this paper, we shall focus on the formal aspect of quantum mechanical histories. We do not find necessary to commit to any particular interpretation: we only assume that all physical information about probabilities and interference of histories is encoded in the decoherence functional, something very explicitly shown by Gell-Mann and Hartle. It is not our aim to insist on how this information can be extracted: both the logic of consistent sets and the Copenhagen stance provide ways of doing this. Perhaps these ways do not exhaust the physical content of the theory -we have argued this in reference [7], but each of them is separately adequate to account for all minimal predictions of standard quantum theory.

We view the histories formalism simply as the covariant version of quantum theory. As such, it incorporates features of the covariant formulation of both classical mechanics and probability theory. But interference of probabilities highlights its quantum nature, and for this reason we shall pay particular attention to the structure of the decoherence functional.

\section{I.2.2 Temporal logic histories}

We shall work in the context of temporal logic histories. This is a scheme initiated by Isham [8, 9]: its main point is that the quantum logic is preserved in the histories theory if we represent a history proposition $\left(\alpha_{t_{1}}, \ldots, \alpha_{t_{n}}\right)$ by a projection operator on a tensor product of the Hilbert spaces of the canonical theory $\mathcal{V}=\otimes_{i} H_{t_{i}}$. This history proposition will then be written as $\alpha=\alpha_{t_{1}} \otimes$ $\ldots \otimes \alpha_{t_{n}}$. This construction is completely analogous to the construction of the space of classical histories as a Cartesian product of single-time sample spaces.

In this formulation a self-adjoint operator on $\mathcal{V}$ represents a history observable for the physical system. As in any covariant theory, more general observables can be defined. They correspond to time-averages and include, for instance, an action operator.

One of the great strengths of this formalism is found in its temporal structure. It was shown by Savvidou [10, 11], that one can mathematically distinguish between two qualities of time: its partial ordering properties ( the notion of before and after) and its status as a dynamical parameter in the equations of motion.

To see this, examine equation (1.1) for the class operator $C_{\alpha}$ entering the expression for the decoherence functional. There, time appears in two places: as an index of the projectors $\hat{\alpha}_{t}$ and as the argument of the unitary operators $\hat{U}(t)$. In its former status, it is purely a kinematical parameter labelling the moment upon which a proposition is asserted. Its function is to determine the order upon which propositions are asserted, in the sense that if $t_{1} \leq t_{2}$ the projection 
operator $\hat{\alpha}_{t_{1}}$ will appear on the left of the operator $\hat{\alpha}_{t_{2}}$ in the equation (1.1) for $\hat{C}_{\alpha}$. In its latter status as the argument of the unitary operators, time is the parameter of the Heisenberg-type evolution. It, thereby, implements the dynamics of the system.

These two roles of the time parameter are completely disentangled, when we view histories in the tensor product Hilbert space $\mathcal{V}=\otimes_{t} H_{t}$. This is an intriguing property, since it allows us to mathematically distinguish between the two conceptually distinct roles by which time appears in physical theories: in the form of a causal structure, that determines the order of events and in the form of the parameter by which change is effected in a physical system.

Indeed in the histories formalism there appear two mathematically distinct laws of time transformation. The partial ordering aspect of time is manifested in translations of the form $H_{t} \rightarrow H_{t+a}$, by which a property asserted at time $t$ is translated to the same property at time $t+a$. At the continuum limit these transformations are generated by the kinematical part of an action operator. Dynamical time transformations are equivalent to a separate unitary transformation for each single-time Hilbert space $H_{t}$. They correspond to the Hamiltonian part of the action.

This distinguishing presence of two laws of time transformation is an important physical principle, that will provide an guideline for the construction of history theories, in the case where the canonical formalism does not provide sufficient insight. In retrospect, one can see that this distinction is present in all physical theories that are formulated in a covariant (histories) fashion [11].

\section{I.3 This paper}

Since our aim is to show how histories provide a covariant formulation of quantum theory, we need to go beyond the discrete-time description that is usually effected: time, in physics, is a continuum. Continuous-time histories have been introduced in 12] and further studied in [13, 10, 14, 15. This work relied on the use of a Fock space for the history Hilbert space, which is only justified if the Hamiltonian is quadratic.

The first aim of this paper is, therefore, to explore the nature of continuous time in this framework. In particular, we highlight the structures that arise in the probability assignment. The analogy with stochastic processes is quite helpful in this regard, both at a conceptual and at a technical level.

In section 2 we explain how a continuous-time Hilbert space with physically interesting observables can be constructed. We then analyze the decoherence functional: we show that it can be decomposed in a way that respects the two laws of time transformation. In fact, its components reflect the distinction between the geometric and the dynamical phase of canonical quantum theory [16]. Finally, we discuss the time-reversal transformations, which are substantially different from the ones of standard quantum theory.

In section 3 we study the phase space structure of histories. This is incorporated in the quantum theory through the use of the history group, the history analogue of the canonical group. The history Hilbert space carries one of its 
representations. This allows the identification of self-adjoint operators in this Hilbert space with objects that have a classical phase space analogue. We explain, how one can construct such representations from the knowledge of the canonical theory.

The analogy with classical probability suggests that one should treat the decoherence functional as the quantum analogue of a classical probability measure. In this sense its "Fourier transform" yields the analogue of the generating function of classical probability: this is the closed-time-path (CTP) generating functional, first introduced by Schwinger [17. We show how to construct this object for phase space histories. This construction suggests that the Wigner transform is of relevance: it enables us to write the decoherence functional as a complex-valued measure on the space of phase space paths and provides a picture of quantum theory that makes reference only to classical objects. One of the merits of this construction is that it provides an algorithmic procedure for passing into the classical limit of generic quantum theories.

In the final section we review our results. We argue that the formalism is flexible enough to accommodate a large number of applications in different fields. In particular, we stress the importance of our results as part of the developing continuous-time histories programme.

Overall, our attitude is to highlight similarities of structures between the histories formalism and more familiar physical formalisms, such as stochastic processes or canonical quantum theory.

\section{I.3.1 Notation}

In the following, our expressions will make reference to two different types of Hilbert space: canonical ones and history ones. We adopt the following conventions: we will use the braket notation to denote vectors of both types of Hilbert space. But we will insert a subscript in the ket denoting a canonical Hilbert space. Hence, for instance, $\left|\psi_{t}\right\rangle_{H_{t}}$ will denote a vector on the canonical Hilbert space $H_{t}$, while $|\psi\rangle$ will denote a vector on a history Hilbert space $\mathcal{V}$.

Also, operators on canonical Hilbert spaces will carry a hat, while the history ones will be unhatted.

As already seen in the introduction, we use the same symbol (small Greek letters) to denote both a proposition and the projector that represents it. We let the meaning be determined by the context.

The notation in section 3 is more complicated, because of the many spaces involved. Points of the (linear) phase space $\Gamma$ will be denoted as $(q, p)$. But

there is also the space $\tilde{\Gamma}$, which is the vector space dual of $\Gamma$ and (if $\Gamma$ is a Hilbert space) isomorphic to it. Points on this space will be denoted as $(\chi, \xi)$ : they correspond to elements of the canonical group or labels of coherent state vectors. The latter will often be denoted as $|z\rangle$. The inner product in these spaces, will be denoted by a dot: we will write invariably $q \cdot p$ or $q \cdot \xi$, without reference to whether the arguments are elements of $\Gamma$ or $\tilde{\Gamma}$. In fact, we shall mostly ignore their distinction.

Paths on $\Gamma$ will be denoted as $(q, p)(\cdot)$, or $t \rightarrow\left(q_{t}, p_{t}\right)$, or simply $\gamma$. Paths 
on $\tilde{\Gamma}$, corresponding to coherent state histories by $(\chi, \xi)(\cdot)$, or $t \rightarrow\left(\chi_{t}, \xi_{t}\right)$, or simply $z(\cdot)$. We will write $(q, \xi)=\int d \mu(t) q_{t} \xi(t)$. When we want to emphasize that $\xi$ also acts as a smearing function on $q_{t}$ we will denote the same object as $q_{\xi}$.

\section{Continuous-time histories}

\section{II.1 The basic structure}

The temporal logic histories scheme is based on ideas from quantum logic. It seeks to represent the set of all history propositions about a physical system with elements of a lattice, that contains the information about the temporal structure 8].

Let us denote by $T$ the set of all instants of time (this can be either discrete, or the real line $\mathbf{R}$ or a subset of $\mathbf{R}$ ). Standard quantum theory is recovered, when we consider that history propositions correspond to projection operators on a Hilbert space $\mathcal{V}$, given by the tensor product $\otimes_{t \in T} H_{t}$. Here $H_{t}$ is a copy of the Hilbert space of the canonical theory indexed by $t$.

Self- adjoint operators on this Hilbert space correspond to history observables.

As an example, let us consider the case where $T$ is a finite set. Let $\hat{A}$ be a bounded operator on the Hilbert space $H$ of the canonical theory, and let us denote by $\hat{A}_{t}$ its copy on a Hilbert space $H_{t}$. Then we can define the product operator $\otimes_{t \in T} \hat{A}_{t}$ on $\mathcal{V}$.

If $\hat{A}_{t}$ is unit everywhere, but a single point $t \in T$, then we shall denote the product operator on $\mathcal{V}$ as $A_{t}$. If $f: T \rightarrow \mathbf{R}$ then we can define the time-averaged operator $A_{f}$ as

$$
A_{f}=\sum_{t \in T} f(t) A_{t}
$$

It corresponds to the average in time of the family $t \rightarrow \hat{A}_{t}$, with a weight given by the function $f$. We can easily verify the following identity. If $\hat{A}$ is a self-adjoint operator on $H$, then its time-averaged counterpart on $\mathcal{V}$ satisfies

$$
e^{i A_{f} s}=\otimes_{t \in T} e^{i \hat{A} f(t) s}
$$

We shall use this identity to define time-averaged operators in the continuoustime case.

Note also that for the case of projection operators, a map $\hat{\alpha} \rightarrow \alpha_{t}$ provides a continuous embedding of the lattice of propositions at a single moment of time to the lattice of history propositions.

The probabilistic content of the theory is contained in the decoherence functional. This is assumed to satisfy the following conditions

$$
\begin{aligned}
d(1,1) & =1 \\
d(\alpha, \beta) & =d^{*}(\beta, \alpha)
\end{aligned}
$$




$$
\begin{aligned}
d(0, \alpha) & =0 \\
d\left(\alpha+\beta, \alpha^{\prime}\right) & =d\left(\alpha, \alpha^{\prime}\right)+d\left(\beta, \alpha^{\prime}\right) \\
d(\alpha, \alpha) & \geq 0
\end{aligned}
$$

In general, there exists a class of operators $X$ on $\mathcal{V} \otimes \mathcal{V}$, such that a decoherence functional can be written as 18, 19]

$$
d(\alpha, \beta)=\operatorname{Tr}_{\mathcal{V} \otimes \mathcal{V}}(X \alpha \otimes \beta)
$$

When the space $T$ is finite, the construction of the tensor product Hilbert space is straightforward and equation (1.4) can be used to construct the decoherence functional. The question arises then, how one deals with continuous time. This is the case when $T$ is a closed subset of the real line. For particular systems the construction of such Hilbert spaces has been carried in [12]. For more general cases, we believe it is instructive to look at the analogous situation in the classical setting.

\section{II.2 Classical stochastic processes}

Let us assume we have a classical system that at a moment of time is described by a sample space $\Omega$. Let us also consider the space $T$ of time instants to be a closed subset of the real line, say $[0, a]$. The space of histories $\Pi$ is then some suitable subset of the set $\Omega^{T}$ of all measurable maps $\gamma: T \rightarrow \Omega$. If $\Omega$ is a vector space one can define a norm on $\Omega^{T}$, and take as $\Pi$ the subspace of $\Omega^{T}$, that contains paths with finite norm.

A function $f$ on $\Omega$ defines a family of functions $F_{t}$ on $\Pi$ by

$$
F_{t}(\gamma)=f(\gamma(t))
$$

As a stochastic process, we usually define a triplet consisting of the space $\Pi$, a family $F_{t}$ and a measure $d \mu$ on $\Pi$. The issue is how to construct physically interesting measures on $\Pi$, which is an infinite dimensional function space.

This is effected as follows: Let $d x$ be for brevity a natural integration measure on $\Omega$ (say a Lebesque measure). Let $T=\left[t_{0}, t_{f}\right]$ be an interval and let us also consider a discretization $I=\left\{t_{0}, t_{1}, \ldots t_{n}=t_{f}\right\}$ of $T$. Then define the space of discrete time histories $\Omega^{I}=\times_{t_{j} \in I} \Omega_{t_{j}}$, which is a finite dimensional manifold. This admits the measure $\prod_{t_{j} \in I} d x_{t_{j}}$. Any probability distribution $p_{I}\left(x_{t_{1}}, \ldots, x_{t_{n}}\right)$ on $\Omega^{I}$ defines a measure $d \mu(x)=p_{I}\left(x_{t_{1}}, \ldots x_{t_{n}}\right) \prod_{t_{j} \in I} d x_{t_{j}}$.

As we consider all possible discretizations $I$ of $T$, we can encode a choice of probability measure for each discretization in a hierarchy of positive functions

$$
\begin{aligned}
& p_{1}(x, t) \\
& p_{2}\left(x_{1}, t_{1} ; x_{2}, t_{2}\right) \\
& \ldots \\
& p_{n}\left(x_{1}, t_{1} ; \ldots ; x_{n}, t_{n}\right)
\end{aligned}
$$


These have to be symmetric with respect to interchange of their $(x, t)$ arguments.

Now, the fundamental theorem of Kolmogorov asserts the following: If a hierarchy of functions as above, satisfies the additivity condition:

$$
\int d x_{n} p_{n}\left(x_{1}, t_{1} ; \ldots ; x_{n-1}, t_{n-1} ; x_{n}, t_{n}\right)=p_{n-1}\left(x_{1}, t_{1} ; \ldots ; x_{n-1}, t_{n-1}\right)
$$

then there exists an essentially unique probability measure $d \mu(\cdot)$ on $\Omega^{T}$ such that it gives the correct discrete time probability measures, i.e. for each partition $I$ , $j_{I}^{*} d \mu=d \mu_{I}$, where $j_{I}$ is the natural injection map $j_{I}: I \rightarrow T$.

Kolmogorov's proof is standard textbook material and is one instance of a general categorical construction of taking the inductive limit. The essential point in the proof is the fact that $j_{I}$ is a measurable map (with respect to the Borel sets of $T$ ) and as such it respects the measurable structure in the definition of $d \mu$.

Hence a probability measure is defined for continuous time, while making reference only to discrete time expressions. This is the theorem that we will try to employ, in order to construct the decoherence functional for continuous- time histories.

\section{II.3 The continuum limit}

\section{II.3.1 The Hilbert space}

The first objective would be to define a suitable version of the Hilbert space $\mathcal{V}=\otimes_{t \in T} H_{t}$. This expression cannot be taken literally, for a continuous tensor product of Hilbert spaces leads to a non-separable Hilbert space. What we will do is a generalization of an idea that has been applied to "continuous tensor products" of Fock spaces [12].

Consider the space $B(T, H)$ of continuous maps $|\psi(\cdot)\rangle$ from $T$ to $H$. In fact, we can start our construction considering only measurable maps. But since we will later want to define Stieljes integrals, we should impose the restriction that the maps are of bounded variation, i.e. they satisfy the following property:

For any finite discretization of $T:\left\{t_{0} \leq t_{1}, \leq \ldots \leq t_{i} \leq \ldots \leq t_{n}\right\}$, the sum $\sum_{i=1}^{n}\left\|\psi_{t_{i}}-\psi_{t_{i-1}}\right\|_{H}$ is finite.

Assume that $T$ has a measure $d \mu(t)$, which in the standard case should be taken as $\frac{d t}{\tau}$. Here $\tau$ is a time parameter that makes the measure dimensionless. If $T$ is compact it can be used to normalize the measure $\mu(T)=1$.

Then define the inner product

$$
\langle\psi(\cdot) \mid \phi(\cdot)\rangle=\prod_{d \mu(t)}\left\langle\psi_{t} \mid \phi_{t}\right\rangle:=\exp \left(\int d \mu(t) \log \left[\left\langle\psi_{t} \mid \phi_{t}\right\rangle_{H_{t}}\right]\right),
$$

where it is understood that the inner product vanishes if $\left\langle\psi_{t} \mid \phi_{t}\right\rangle_{H_{t}}=0$ in a subset of $T$ that is not of measure zero, and that the logarithm takes values on the principal branch. 
This space has then a norm $\|\psi(\cdot)\|=(\langle\psi(\cdot) \mid \psi(\cdot)\rangle)^{1 / 2}$. We identify two elements $\psi_{1}(\cdot), \psi_{2}(\cdot)$ of $B(T, H)$, if $\left\|\psi_{1}(\cdot)-\psi_{2}(\cdot)\right\|=0$. This identification makes the resulting Hilbert space separable.

Let us, suggestively, denote the vector space we obtained after identification, as $\times_{t \in T} H_{t}$. To construct $\otimes_{t \in T} H_{t}$ we consider the space of all formal linear combinations $\sum_{i} c_{i}\left|\psi_{i}(\cdot)\right\rangle$. Here $i$ runs over a finite set, $c_{i} \in \mathbf{C}$, and $\left\{\left|\psi_{i}(\cdot)\right\rangle\right\}$ is a finite set of vectors of $\times_{t \in T} H_{t}$. On the space of these formal linear combinations we define the inner product as

$$
\sum_{i} \bar{c}_{i}{ }_{i} c_{i}\left\langle\psi_{i}^{\prime}(\cdot) \mid \psi_{i}(\cdot)\right\rangle
$$

and close this space with respect to the norm. We have thus defined a Hilbert space $\otimes_{t \in T} H_{t}$. Note that the time parameter $\tau$ enters explicitly into the definition.

The vectors $|\psi(\cdot)\rangle$ form a total set of $\otimes_{t \in T} H_{t}$. As such, we can define operators on the history Hilbert space by their action on these vectors.

Some properties of this construction are easy to see. For instance

$$
\otimes_{t} e^{\lambda_{t}}\left|\psi_{t}\right\rangle_{H_{t}}=e^{\int d \mu(t) \lambda(t)}|\psi(\cdot)\rangle .
$$

Also, if $T_{1}$ and $T_{2}$ are two disjoint subsets of $\mathbf{R}$ with non-zero measure, then

$$
\otimes_{t \in T_{1} \cup T_{2}} H_{t}=\left(\otimes_{t \in T_{1}} H_{t}\right) \otimes\left(\otimes_{t \in T_{2}} H_{t}\right) .
$$

\section{II.3.2 Time-averaged observables}

Let $\hat{A}_{t}$ is a continuous family of bounded operators on $H$ indexed by $t$. Then one can define the product operator $\otimes_{t \in T} \hat{A}_{t}$ by its action on $|\psi(\cdot)\rangle$

$$
\left(\otimes_{t \in T} \hat{A}_{t}\right)|\psi(\cdot)\rangle=\otimes_{t \in T}\left(\hat{A}_{t}\left|\psi_{t}\right\rangle_{H_{t}}\right) .
$$

This definition is extended by linearity to the whole Hilbert space. However, one has to restrict the families $t \rightarrow \hat{A}_{t}$. We have

$$
\begin{array}{r}
\|\left(\otimes_{t \in T} \hat{A}_{t}\right)|\psi(\cdot)\rangle \|^{2}=\exp \left(\int d \mu(t) \log \left(\left\langle\psi_{t}\left|\hat{A}_{t}^{2}\right| \psi_{t}\right\rangle_{H_{t}}\right)\right. \\
\leq \exp \left(\int d \mu(t) \log \left(\left\|A_{t}\right\|^{2}\left\langle\psi_{t} \mid \psi_{t}\right\rangle_{H_{t}}\right)\right)=e^{\int d \mu(t) \log \left(\left\|\hat{A}_{t}\right\|^{2}\right)}\langle\psi(\cdot) \mid \psi(\cdot)\rangle,
\end{array}
$$

hence one has to demand that $\int d \mu(t) \log \left(\left\|\hat{A}_{t}\right\|^{2}\right) \leq \infty$. If $T$ is a compact subset of $\mathbf{R}$, this holds automatically provided the map $t \rightarrow\left\|\hat{A}_{t}\right\|$ is measurable. If $T$ is non-compact, e.g. the whole of $\mathbf{R}$, the right hand side is not finite and one has to additionally demand that $\hat{A}_{t}=1$ outside some compact subset of $\mathbf{R}$, or that $\left\|\hat{A}_{t}-\hat{1}\right\|_{H}$ falls to zero sufficiently rapidly.

It is easy to see that

$$
\operatorname{Tr}_{\mathcal{V}}\left(\otimes_{t \in T} \hat{A}_{t}\right)=\prod_{t \in T}\left(\operatorname{Tr}_{H_{t}} \hat{A}_{t}\right)=\exp \left(\int d \mu(t) \log \operatorname{Tr}_{H_{t}} \hat{A}_{t}\right) .
$$


Having defined the product operators we can define time-averaged observables, by exploiting equation (2.2). Let $\hat{A}$ be a bounded self-adjoint operator on $H$. We can write the family $t \rightarrow \hat{U}_{t}(s)=e^{i \hat{A} f(t) s}$ of unitary operators and construct the product operator $U_{f}(s)=\otimes_{t \in T} \hat{U}_{t}(s)$. This is well-defined if $f(t) \neq 0$ only within a compact subset of $\mathbf{R}$ and corresponds to an one-parameter group of unitary operators on $\mathcal{V}$. By Stone's theorem, if the matrix elements of this operator are continuous functions of $s$ at $s=0$, there exists a self-adjoint operator $A_{f}$ such that $U_{f}(s)=e^{i A_{f} s}$.

It is easy to check, that

$$
\left\langle\phi(\cdot)\left|U_{f}(s)\right| \psi(\cdot)\right\rangle=\exp \left(\int d \mu(t) \log \left(\left\langle\phi_{t}\left|e^{i \hat{A} f(t) s}\right| \psi_{t}\right\rangle\right)\right)
$$

is a continuous function of $s$ at $s=0$, when the operator $\hat{A}$ is bounded. Thus, given suitable functions $f$, a self-adjoint operator representing the time average of $\hat{A}$ is well defined on $\mathcal{V}$.

\section{II.3.3 Unbounded operators}

The construction of time-averaged counterparts to unbounded operators on $H$ is more complicated. From equation (2.15) we see that even if the matrix elements $\left\langle\psi_{t}\left|e^{i \hat{A} f(t) s}\right| \phi_{t}\right\rangle_{H_{t}}$ are continuous functions of $s$, there is no guarantee that so will be the integral.

Also if $\hat{A}$ is unbounded, there exist vectors $\left|\psi_{t}\right\rangle$, for which the action of $\hat{A}_{t}$ is not defined, hence one cannot write $\left|\left\langle\phi_{t}\left|e^{i \hat{A} f(t) s}\right| \psi_{t}\right\rangle\right| \leq c|s|$, which would be sufficient to prove continuity. There is no guarantee that the time-average of an unbounded operator is definable.

This is unfortunate, because in physical situations we are interested in operators like position, or momentum, or the Hamiltonian, that are typically unbounded. This failure is due to the fact that the Hilbert space $\otimes_{t \in T} H_{t}$ is still very large. In concrete physical situations one should identify the histories Hilbert space $\mathcal{V}$ with a closed linear subspace of $\otimes_{t \in T} H_{t}$.

One has to choose this closed linear subspace, in such a way that the tensor product structure is preserved. The simplest way is to restrict the set of vectors that can be used to construct the "paths" $|\psi(\cdot)\rangle$ to a subset $\mathcal{L}$ of $H$. This set $\mathcal{L}$ has to be sufficiently large to be able to capture all physical information from $H$ (it cannot be a subspace of $H$ ), but small enough to allow interesting operators to be definable on the history Hilbert space. A good choice for $\mathcal{L}$ is an overcomplete and continuous family of vectors, like the coherent states.

Having chosen $\mathcal{L}$, the construction proceeds as before, only we substitute $B(T, H)$ with $B(T, \mathcal{L})$ : the space of all continuous maps from $T$ to $\mathcal{L}$. It is easy to check that the resulting Hilbert space is a closed linear subspace of $\otimes_{t \in T} H_{t}$.

If we demand that a particular unbounded operator $A$ exists (time-averaged) in our Hilbert space, it would be necessary to take $\mathcal{L}$ consisting of vectors in the domain of $A$. In that case the matrix elements (2.15) would be a continuous function of $s$ and (by Stone's theorem) $A_{f}$ would exist. We shall see how this 
construction works in more detail, in section 3. In this section, we shall work with the larger Hilbert space $\otimes_{t \in T} H_{t}$. All results we obtain will be valid for any of its physically relevant subspaces.

\section{II.3.4 The decoherence functional}

If $T$ is compact, one can choose $A_{t}=A$ for all $t$ and therefore interpret $A_{f}$ as the time average of the quantity associated to $A$. But, if we try to define an operator on $\mathcal{V}$, that corresponds to an observable at a sharp moment of time, we run into problems. Since a point in the real axis is of measure zero, an observable defined at a sharp moment of time can exist only if we can take $f$ to be a delta function. This is unacceptable in our construction. We conclude that one cannot embed continuously the lattice of single-time propositions into the lattice of history propositions, in the case of continuous time.

Let us now examine the possibility of defining a decoherence functional for continuous-time histories as a continuous limit of the discrete-time expression (1.4). Let us assume a partition $I=\left\{t_{1}, \ldots, t_{n}\right\}$ of an interval $T$ of the real line and a proposition $\alpha=\alpha_{t_{1}} \otimes \ldots \otimes \alpha_{t_{n}}$ that is a projector operator on $H^{I}=\otimes_{t_{i} \in I} H_{t_{i}}$. Then one can construct the class operator $\hat{C}_{\alpha}$ defined on one one copy of $H$ as in equation (1.1). The value of decoherence functional $d_{I, I^{\prime}}$ between a history on $H^{I}$ and another on some other discrete-time Hilbert space $H^{I^{\prime}}$ is given by equation (1.4).

The aim is to generalise Kolmogorov's theorem in this histories setting. We want to construct a bilinear, hermitian, additive map on the space $P(\mathcal{V}) \times P(\mathcal{V})$ (by $P(H)$ we mean the lattice of projectors on the Hilbert space $H$ ). If we consider then a pair of discretisations $I$ and $I^{\prime}$ of $T$, we can costruct the Hilbert spaces $H^{I}$ and $H^{I^{\prime}}$. The point is whether there exist an injection map $j_{I, I^{\prime}}$ : $H^{I} \times H^{I^{\prime}} \rightarrow \mathcal{V} \times \mathcal{V}$; if this exists and preserves the lattice structures then Kolmogorov's proof goes through and the decoherence functional $d$ on $H^{T}$ exists as an inductive limit of the decoherence functional defined on $H^{I} \times H^{I^{\prime}}$ for all choices of $I$ and $I^{\prime}$. We would also have $d_{I, I^{\prime}}=j_{I, I^{\prime}}^{*} d_{T}$.

For the map to be lattice-preserving it would have to be continuous. But, we showed earlier, that this cannot be true for a single moment of time. The map $j_{I, I^{\prime}}$ might be continuous in the weak topology, but this is insufficient to define an order preserving map. Recall that the continuity of the Hilbert space enters in a decisive point in the definition of the lattice of propositions: a projection operator corresponds to a closed linear subspace. Hence Kolmogorov's theorem does not go through in this case.

But if we restrict to an Abelian sublattice, (for instance, to propositions about position) the map $j_{I, I^{\prime}}$ does not need to be a continuous, linear map, but simply a measurable map from the spectra of the corresponding operators $\mathbf{R}^{I} \times \mathbf{R}^{I^{\prime}}$ to $\mathbf{R}^{T} \times \mathbf{R}^{T}$. This clearly exists; it is the same as in the case of classical probability theory.

We therefore conclude that one cannot write the decoherence functional for continuous time, as a limit of discrete-time ones, unless one restricts to Abelian subalgebras. We might have a continuous- time decoherence functional for each 
subalgebra, but not one defined on the whole of $P(\mathcal{V})$. We shall return to this issue again and propose two different ways, by which the decoherence functional can be defined.

\section{II.4 The structure of the decoherence functional}

The presence of two laws of time transformation is an important structural feature of history theories. In this section, we shall show how they are manifested in the probability assignment. We shall see that the decoherence functional for discrete time can be written in such a way, that these two notions of time are distinctly represented. This is a feature, that in the canonical theory is reflected in the distinction between geometric and dynamical phase [16]. And this feature we shall attempt to generalise in the continuous-time case.

For simplicity we shall consider a special class of decoherence functionals. They are of the type (1.4), but with $\hat{\rho}_{0}$ corresponding to a pure state. This means that we can absorb the projector into the initial state as part of the definition of each history and as such write the decoherence functional in the form

$$
d(\alpha, \beta)=\operatorname{Tr}_{H}\left(\hat{C}_{\alpha}^{\dagger} \hat{C}_{\beta}\right) .
$$

Clearly one of the single-time projectors has to be trace-class if the above expression is to be finite. Equation (2.16) can be written as 18

$$
d(\alpha, \beta)=\operatorname{Tr}_{H \otimes H}\left(Z \hat{C}_{\alpha}^{\dagger} \otimes \hat{C}_{\beta}\right),
$$

where $Z$ is an operator on $H \otimes H$ given by

$$
Z(|i\rangle \otimes|j\rangle)=|j\rangle \otimes|i\rangle .
$$

One can write $Z=\sum_{r s} \hat{A}^{r s} \otimes \hat{A}^{\dagger r s}$ in terms of a basis on $\mathrm{H}$, where $\hat{A}^{r s}$ is an operator on $H$ with matrix elements

$$
\left\langle k\left|\hat{A}^{r s}\right| i\right\rangle=\delta_{k s} \delta_{r i} .
$$

Let us now assume that both histories are defined in the same instants of time $t_{0}, t_{1}, \ldots t_{n}$. Let us for simplicity take $t_{0}=0$. The corresponding history Hilbert space is then $\mathcal{V}=\otimes_{i} H_{t_{i}}$.

Let us also write the boundary Hilbert space $\partial \mathcal{V}=H_{t_{0}} \otimes H_{t_{n}}$. The indices $r s$ of the operators $\hat{A}^{r s}$ are then indices corresponding to $\partial \mathcal{V}$. It is easy to verify that the expression (2.17) can be written as a trace over the boundary Hilbert space 14.

$$
d(\alpha, \beta)=\operatorname{Tr}_{\partial \mathcal{V}}\left(c(\alpha) c^{\dagger}(\beta)\right),
$$

where $c(\alpha)$ is an operator on $\partial \mathcal{V}$ defined by

$$
c(\alpha)=\operatorname{Tr}_{H} \hat{A} \hat{C}_{\alpha}^{\dagger} .
$$


$\hat{A}$ denotes here a map from $H$ to $\mathcal{V}$. It is easy now to write $c(\alpha)$ as a trace over the history Hilbert space, through the introduction of the unitary operator $\mathcal{S}$ on $\mathcal{V}$

$$
\mathcal{S}\left|v_{t_{0}}\right\rangle\left|v_{t_{2}}\right\rangle \ldots\left|v_{t_{n}}\right\rangle=\left|v_{t_{n}}\right\rangle\left|v_{t_{0}}\right\rangle \ldots\left|v_{t_{n-1}}\right\rangle .
$$

Indeed, since $\alpha=\hat{\alpha}_{t_{0}} \otimes \hat{\alpha}_{t_{1}} \otimes \ldots \otimes \hat{\alpha}_{t_{n}}$, we can write

$$
c(\alpha)=\operatorname{Tr}_{\mathcal{V}}\left(\mathcal{A S U}^{\dagger} \alpha \mathcal{U}\right),
$$

where

$$
\begin{array}{r}
\mathcal{U}=\hat{U}\left(t_{0}\right) \otimes \hat{U}\left(t_{1}\right) \ldots \otimes \hat{U}\left(t_{n}\right), \\
\mathcal{A}=\hat{A} \otimes 1 \otimes \ldots \otimes 1 .
\end{array}
$$

This accomplishes the task of writing the decoherence functional in such a way as the two different notions of time are made manifest. The operator $\mathcal{U}$ clearly contains the dynamics. The operator $\mathcal{S}$ induces a transformation that takes from a single-time Hilbert space to the next one. Finally, the operators $A$ incorporate the information about the beginning and the end of the interval. Had we kept the initial density matrix, $\hat{A}$ would explicitly depend upon it. In that case the analogue of equation (2.19) would be

$$
\left\langle k\left|\hat{A}^{r s}\right| i\right\rangle=\delta_{k s}\left(\rho_{0}^{1 / 2}\right)_{r i} .
$$

\section{II.5 The continuum limit}

Let us now examine whether one can construct these operators in the continuoustime Hilbert space $\otimes_{t \in T} H_{t}$, which we defined earlier.

The operator $\mathcal{U}$ is relatively easy to define. It would act on a vector $|\psi(\cdot)\rangle$ as

$$
\mathcal{U}|\psi(\cdot)\rangle=\otimes_{t \in T}\left(e^{-i \hat{H} t}\left|\psi_{t}\right\rangle_{H_{t}}\right) .
$$

This would have as matrix elements

$$
\langle\phi(\cdot)|\mathcal{U}| \psi(\cdot)\rangle=\exp \left(\int d \mu(t) \log \left\langle\phi_{t}\left|e^{-i \hat{H} t}\right| \psi_{t}\right\rangle\right) .
$$

According to our previous analysis this is a genuine unitary operator on $\mathcal{V}$.

\section{II.5.1 The geometric phase}

The operator $\mathcal{S}$ has an important geometric significance. It incorporates information about the geometric phase [20, 21] that is associated to a history. To see this, one has first to recall that a Hilbert space $H$ is a line bundle over the projective Hilbert space $P H$, i.e. the equivalence class of all vectors that differ by a multiplication with a complex number. We shall denote an element of $P H$ 
as $[\psi]$. The inner product on $H$ inherits two important geometric structures on $P H$ : a metric

$$
d s^{2}=\| d|\psi\rangle \|^{2}-|\langle\psi|d| \psi\rangle|^{2},
$$

and a $U(1)$ connection

$$
A=-i\langle\psi|d| \psi\rangle \text {. }
$$

When a point of $P H$ evolves along a loop $\gamma$, its total phase change consists of a piece that depends upon the dynamics and a piece that is essentially the holonomy of the connection $A$ [22, 23]. This is known as the Berry phase and equals

$$
e^{i \theta_{g}[\gamma]}=e^{i \int_{\gamma} A}=\exp \left(\int\langle\psi|d| \psi\rangle\right)
$$

The geometric phase can also be defined for open paths. The trick is that any path on the projective Hilbert space can be closed by joining its endpoints with a geodesic, with respect to the natural metric. The geometric phase of the loop thus constructed is then defined to equal the geometric phase associated to the open path. Hence if $\gamma=[\psi(\cdot)]$ is a path on $P H$ its associated geometric phase can be found 24]

$$
e^{i \theta_{g}[\gamma]}=\exp \left(\int_{t_{i}}^{t_{f}} d t\langle\psi(t) \mid \dot{\psi}(t)\rangle\right)\left\langle\psi_{i} \mid \psi_{f}\right\rangle
$$

This expression is defined only if the endpoints are not orthogonal.

Now let us consider a discretized approximation to an element $|\psi(\cdot)\rangle$ of $\mathcal{V}$. Let us write therefore,

$$
\alpha=\otimes_{t_{j}}\left|\psi_{t_{j}}\right\rangle\left\langle\psi_{s_{j}}\right|
$$

where $\left|\psi_{t_{j}}\right\rangle$ are normalized vectors on $H_{t_{j}}$.

We then calculate

$$
\operatorname{Tr}(\mathcal{S} \alpha)=\left\langle\psi_{t_{0}} \mid \psi_{t_{n}}\right\rangle\left\langle\psi_{t_{1}} \mid \psi_{t_{0}}\right\rangle\left\langle\psi_{t_{2}} \mid \psi_{t_{1}}\right\rangle \ldots\left\langle\psi_{t_{n}} \mid \psi_{t_{n-1}}\right\rangle .
$$

Let us then assume that $\max \left|t_{j}-t_{j-1}\right|=\delta t$, and we choose the number of time steps $n$ very large, so that $\delta t \sim O\left(n^{-1}\right)$. Then $\left|\psi_{t_{j}}\right\rangle$ approximates a path $[\psi(t)]$ on $\mathrm{PH}$. Writing formally $\alpha_{\psi(\cdot)}$ for the projector we get

$$
\begin{array}{r}
\log \operatorname{Tr}\left(\mathcal{S} \alpha_{\psi(\cdot)}\right)=\log \left\langle\psi_{t_{0}} \mid \psi_{t_{n}}\right\rangle+\sum_{i=1}^{n} \log \left\langle\psi_{t_{i}} \mid \psi_{t_{i-1}}\right\rangle \\
=\log \left\langle\psi_{t_{0}} \mid \psi_{t_{n}}\right\rangle+\sum_{i=1}^{n} \log \left(1-\left\langle\psi_{t_{i}} \mid \psi_{t_{i}}-\psi_{t_{i-1}}\right\rangle\right),
\end{array}
$$

which in the limit of large $n$ yields

$$
\log \operatorname{Tr}\left(\mathcal{S} \alpha_{\psi(\cdot)}\right)=\log \left\langle\psi_{t_{0}} \mid \psi_{t_{n}}\right\rangle-\sum_{i=1}^{n}\left\langle\psi_{t_{i}} \mid \psi_{t_{i}}-\psi_{t_{i-1}}\right\rangle+O\left((\delta t)^{2}\right) .
$$


As $\delta t \rightarrow 0$ the sum in the right-hand side converges to a Stieljes integral $-\int_{t_{i}}^{t_{f}} d t\langle\psi(t) \mid \dot{\psi}(t)\rangle$ and hence

$$
\operatorname{Tr}\left(\mathcal{S} \alpha_{\psi(\cdot)}\right)=e^{i \theta_{g}[\psi(\cdot)]}
$$

This is the Berry phase associated to the path $\psi(\cdot)$. This implies that $\mathcal{S}$ exists as an operator on $\mathcal{V}$. Its matrix elements can be defined as

$$
\langle\phi(\cdot)|\mathcal{S}| \psi(\cdot)\rangle=\left\langle\phi\left(t_{0}\right) \mid \psi\left(t_{f}\right)\right\rangle \exp \left(\int\langle\psi(t)|d| \psi(t)\rangle\right),
$$

where the integral in the exponential is of the Stieljes type (rather than of the Lebesque, that was used in the definition of $\otimes_{t \in T} H_{t}$ ). The Stieljes integral is defined for all measurable functions of bounded variation. Hence the matrix elements of $\mathcal{S}$ are finite. This implies, it is a well defined bounded operator and it is easy to check that it remains unitary even in the continuous limit.

\section{II.5.2 Another attempt to construct the decoherence functional}

We have showed that the main operators that form the decoherence functional exist in the continuous limit. Could we then proceed and define a continuoustime decoherence functional from equation (2.20)? The answer is no, at least not straightforwardly. The problem is that the analogue of the maps $\mathcal{A}$ does not exist in the continuous limit. The reason is the same as before: an embedding of single-time Hilbert spaces to the history Hilbert space fails to be continuous.

One has therefore two options. First, it should be noted that an initial and final moment of time is necessary in the decoherence functional, because they incorporate information about the preparation of the system. From an operational viewpoint, one could then say that the specification of an initial state cannot be sharp in time and as such they ought to be incorporated in the decoherence functional by an object that is extended in time. This would imply a generalization of expression (2.23), where the map $\mathcal{A}$ is defined from $\mathcal{V}$ not to $\partial \mathcal{V}$, but to some other Hilbert space, which is associated with a finite time sub-interval of $T$. The introduction of such an operator could provide a construction of a continuous decoherence functional in this case. This would be mathematically well-defined and operationally meaningful, but would diverge from the standard canonical quantum theory. For this reason, we shall not pursue this further in this paper.

An alternative would be to abandon the effort to define a continuous decoherence functional and assume at most weak continuity.

If we assume two one -dimensional projectors $\alpha_{\psi(\cdot)}$ and $\alpha_{\psi(\cdot)}$ we get an expression for the decoherence functional with zero Hamiltonian

$$
\begin{gathered}
d\left(\alpha_{\psi(\cdot)}, \alpha_{\psi(\cdot)}\right)=\left\langle\psi\left(t_{i}\right)\left|\hat{\rho}_{0}\right| \psi\left(t_{i}\right)\right\rangle\left\langle\psi\left(t_{f}\right) \mid \psi\left(t_{f}\right)\right\rangle \\
\exp \left(\int_{t_{i}}^{t_{f}} d t\langle\psi(t) \mid \dot{\psi}(t)\rangle-\int_{t_{i}}^{t_{f}} d t\langle\dot{\psi(t)} \mid \psi(t)\rangle\right) .
\end{gathered}
$$


In the special case where $\hat{\rho}_{0}=\left|\psi\left(t_{i}\right)\right\rangle\left\langle\psi\left(t_{i}\right)|=| \psi\left(t_{i}\right)\right\rangle\left\langle\psi\left(t_{i}\right)\right|$ and $\left|\psi\left(t_{f}\right)\right\rangle\left\langle\psi\left(t_{f}\right)\right|=$ $\left|\psi\left(t_{f}\right)\right\rangle\left\langle\psi\left(t_{f}\right)\right|$ its value is equal to

$$
d\left(\alpha_{\psi(\cdot)}, \alpha_{\psi(\cdot)}\right)=e^{i \theta_{g}[\psi(\cdot), \psi(\cdot)]},
$$

the Berry phase for the loop formed from $\psi(\cdot)$ and $\psi(\cdot)$, since now they have the same endpoints.

More interestingly, when the Hamiltonian is included the decoherence functional becomes

$$
d\left(\alpha_{\psi(\cdot)}, \alpha_{\psi(\cdot)}\right)=\left\langle\psi\left(t_{i}\right)\left|\hat{\rho}_{0}\right| \psi\left(t_{i}\right)\right\rangle\left\langle\psi\left(t_{f}\right)\left|\hat{\rho}_{f}\right| \psi\left(t_{f}\right)\right\rangle e^{i S[\psi(\cdot)]-i S^{*}[\psi(\cdot)]},
$$

where the action is given by the familiar expression (its variation gives the Schrödinger equation)

$$
S[\psi(\cdot)]=\int_{t_{i}}^{t_{f}} d t\left\langle\psi(t)\left|i \frac{d}{d t}-H\right| \psi(t)\right\rangle .
$$

One might then give equation (2.41) as a definition of a decoherence functional for pairs of one-dimensional projectors and then extend this definition by finite addition to projectors with finite trace. But there is no a priori guarantee that one would thus construct an object taking finite values a general projector on $\otimes_{t \in T} H_{t}$. Nonetheless, equation (2.41) highlights the importance of the action as the object relating kinematics, dynamics and the probabilistic structure of quantum theory.

We shall return to the issue of the definition of a continuous-time decoherence functional in section 3.5.3.

\section{II.6 Time reversal}

A symmetry on a history Hilbert space is represented either by a unitary or an antiunitary operator. This has been established by Schreckenberg 25] .

Of particular interest are the time reversal transformations. In discrete time they are defined by [12]

$$
\mathcal{T}\left|v_{t_{1}}\right\rangle\left|v_{t_{2}}\right\rangle \ldots\left|v_{t_{n}}\right\rangle=\left|v_{t_{n}}\right\rangle\left|v_{t_{n-1}}\right\rangle \ldots\left|v_{t_{1}}\right\rangle .
$$

Clearly

$$
\begin{array}{r}
\mathcal{T T}^{\dagger}=1 \\
\mathcal{T S \mathcal { T } ^ { \dagger }}=\mathcal{S}^{\dagger}
\end{array}
$$

Also for the operator $\mathcal{U}$ defined by (2.24) we have

$$
\mathcal{T} \mathcal{U} \mathcal{T}^{\dagger}=e^{-i \hat{H} t_{n}} \otimes \ldots \otimes e^{-i \hat{H} t_{1}}
$$

and when time runs in the full real line

$$
\mathcal{T U} \mathcal{T}^{\dagger}=\mathcal{U}^{\dagger}
$$


Finally for the time inverted projection operators $\alpha^{T}=\mathcal{T} \alpha \mathcal{T}^{\dagger}$ (corresponding to homogeneous histories) we have

$$
d\left(\alpha^{T}, \beta^{T}\right)=\operatorname{Tr}\left(\hat{C}_{\alpha}^{T \dagger} \rho_{0} \hat{C}_{\beta}^{T} \rho_{f}\right)
$$

where $\hat{C}_{\alpha}^{T}=\hat{\alpha}_{t_{1}}\left(t_{n}\right) \ldots \hat{\alpha}_{t_{n}}\left(t_{1}\right)$. In the discrete case this form is not transparent, but when time takes values in all $\mathbf{R}$ the Heisenberg picture operators transform as $\alpha_{t}(t) \rightarrow \alpha_{t}(-t)$ and therefore

$$
d\left(\alpha^{T}, \beta^{T}\right)=d(\beta, \alpha)=[d(\alpha, \beta)]^{*}
$$

Of course this later equation does not hold if the Hamiltonian is time-dependent and the system is not time-homogeneous.

The operator $\mathcal{T}$ is naturally defined also on $\otimes_{t \in \mathbf{R}} H_{t}$

$$
\mathcal{T}|\psi(\cdot)\rangle=\mathcal{T}|\psi(-\cdot)\rangle
$$

It is important to note that the time reversal operator is linear rather than anti - linear as in canonical quantum mechanics. This has again to do with the presence of two laws of time transformations in history theories; here time reversal implemented by $\mathcal{T}$ corresponds to the causal, kinematical properties of time. The time inversion operator of canonical quantum mechanics is obtained by the study of the Schrödinger equation and as such is clearly associated to the dynamical aspect of time.

Of course we can always define an anti-linear time reversal operator in complete analogy with the canonical case; a complex conjugation on $H$ naturally defines a complex conjugation on $\mathcal{V}$. It would act on the Heisenberg picture operators as $\alpha_{t}(t) \rightarrow \alpha_{t}(-t)$.

\section{II.7 Summary}

Let us summarize here the results of this section. We showed how a Hilbert space $\otimes_{t \in T} H_{t}$ for continuous time histories can be constructed and how timeaveraged observables can be defined as operators acting on it. Then we argued that in general we will have to restrict to a particular subset of $\otimes_{t \in T} H_{t}$. We then showed that the decoherence functional cannot be defined as a limiting case of its discrete-time form.

We then analyzed the structure of the decoherence functional. We identified the pieces out of which it is constructed, in light of the two laws of time transformation of history theories, and showed their relation to the dynamical and geometric phase of canonical quantum theory. We discussed a possible way to construct the continuous-time decoherence functional and finally saw how unitary time-reversal transformations are implemented in this scheme.

\section{Phase space histories}

In the previous section we examined the general structure of continuous-time histories, without making any reference to a particular physical system, or class 
of systems. In order to do so, we necessarily have to make reference to a corresponding classical system and seeks to identify operators on the Hilbert space with observables that have a classical analogue. This is, in effect, the quantization procedure. In this section, we will study how the classical phase space structure is manifested in the histories formalism.

We refer the reader to section 1.3.1 for explanation of the notations we will use in this section.

\section{III.1 The canonical group}

\section{III.1.1 The Weyl group}

In quantum theory the information about the corresponding classical theory can be encoded in the canonical group. This is classically identified as a group that acts transitively by canonical transformations on the classical phase space $\Gamma$ [26]. When $\Gamma=\mathbf{R}^{2 n}$ the canonical group is the $(2 n+1)$ - dimensional Weyl group. This is defined whenever the phase space has a vector space structure. It can therefore be infinite dimensional, as in a field theory. For its definition an inner product on $\Gamma$ has to be assumed, so we usually consider $\Gamma$ to be a real Hilbert space.

The Weyl group is generated by $q_{i}, p_{i}, 1$ and has basic Lie algebra relations

$$
\begin{gathered}
\left\{q_{i}, q_{j}\right\}=0 \\
\left\{p_{i}, p_{j}\right\}=0 \\
\left\{q_{i}, p_{j}\right\}=\delta_{i j} .
\end{gathered}
$$

A generator of the Weyl group reads $\chi \cdot p+\xi \cdot q+c$, in terms of the inner product in $\Gamma$, and is labelled by $\left(\chi_{i}, \xi_{i}, c\right)$. The corresponding group element will be denoted as $(\chi, \xi, c)$. The group multiplication law is

$$
\left(\chi_{1}, \xi_{1}, c_{1}\right) \cdot\left(\chi_{2}, \xi_{2}, c_{2}\right)=\left(\chi_{1}+\chi_{2}, \xi_{1}+\xi_{2}, c_{1}+c_{2}+\frac{1}{2}\left(\xi_{1} \cdot \chi_{2}-\xi_{2} \cdot \chi_{1}\right)\right)
$$

When the canonical group has been identified, the Hilbert space of the theory is constructed through the selection of one of its unitary irreducible representations. The criterion for this selection is the existence of self-adjoint operators that correspond to the generators of classical symmetries (e.g. the Hamiltonian, the Lorentz group etc).

\section{III.1.2 Coherent states}

Suppose we have a representation of the canonical group by unitary operators $\hat{U}(g)$ on a Hilbert space. Furthermore, let $\hat{h}$ denote the Hamiltonian of this system and by $|0\rangle_{H}$ the vacuum, i.e. the Hamiltonian's lowest eigenstate. Then we define the coherent states as the vectors

$$
|g\rangle=\hat{U}(g)|0\rangle .
$$


Now consider the equivalence relation on the canonical group defined as $g \sim g^{\prime}$ if $|g\rangle$ and $\left|g^{\prime}\right\rangle$ correspond to the same ray. The phase space $\Gamma$ is identified as the quotient space $G / \sim$ and we can label a coherent state by points $z \in \Gamma$.

Hence the canonical group defines a map $i: \Gamma \rightarrow P H$ as $z \rightarrow|z\rangle$. As we explained $P H$ has a natural metric and a $\mathrm{U}(1)$ bundle structure with a connection. These structures can be pullbacked to $\Gamma$ with $i^{*}$. We have then on $\Gamma$ a $U(1)$ bundle with a connection $A$ given by

$$
A=-i\langle z|d| z\rangle
$$

and a metric

$$
d s^{2}=\left.|| d|z\rangle\right|^{2}-|\langle z|d| z\rangle|^{2},
$$

where $d$ is the exterior derivative on $\Gamma$. The fundamental property of coherent states is that they are an overcomplete basis; i.e. any vector $|\Psi\rangle$ can be written as

$$
|\Psi\rangle=\int d \mu(z) f(z)|z\rangle
$$

in terms of some complex-valued function $f$ on $\Gamma$. Here $d \mu$ denotes some natural measure on $\Gamma$. In $n$ dimensions it is equals $\frac{d^{n} \bar{z} d z}{(2 \pi)^{n}}$. There is also a decomposition of the unity

$$
\int d \mu(z)|z\rangle\langle z|=\hat{1}
$$

If the phase space $\Gamma$ has a vector space structure the canonical group is the Weyl group. Its most usual representation is on $e^{\Gamma_{C}}=\oplus_{n=1}^{\infty}\left(\otimes_{n} H\right)_{S}$, the symmetric Fock space generated by the complex vector space $\Gamma_{C}$, a complexification of $\Gamma$ [27, 28]. On the Fock space there exist the unnormalized coherent states $|\exp z\rangle$ that to each $z \in \Gamma_{C}$ they assign the vector $|\exp z\rangle=\oplus_{n=0}^{\infty} \otimes_{n} z$. The inner product of such states is given by

$$
\left\langle\exp z^{\prime} \mid \exp z\right\rangle=e^{\left(z^{\prime}, z\right)_{C}},
$$

where $(,)_{C}$ denotes an inner product on $\Gamma_{C}$ (its choice depends upon the way $\Gamma$ is complexified). The corresponding normalized states are denoted simply as $|z\rangle$, or $|\chi, \xi\rangle$.

\section{III.1.3 The overlap kernel}

For the finite dimensional Weyl group, the Stone-von Neumann theorem asserts that all irreducible representations are unitarily equivalent to the Fock one. This is not true in infinite dimensions. In this case, the information about the representation is encoded in the coherent states overlap $\left\langle\chi^{\prime} \xi^{\prime} \mid \chi \xi\right\rangle$.

This is determined by the expectation functional $K(\chi, \xi)=\langle 0 \mid \chi, \xi\rangle$ as a consequence of the group combination law

$$
\left\langle\chi^{\prime} \xi^{\prime} \mid \chi \xi\right\rangle=e^{i / 2\left(\chi \cdot \xi^{\prime}-\xi \cdot \chi^{\prime}\right)} K\left(\chi-\chi^{\prime}, \xi-\xi^{\prime}\right) .
$$


The expectation functional suffices to describe the connection and metric structure on phase space. If we write $K=e^{W}$, we find

$$
\begin{array}{r}
A=\xi_{i} d \chi^{i}, \\
d s^{2}=-R e\left(\frac{\partial^{2} W}{\partial \chi^{i} \partial \chi^{j}} d \chi^{i} d \chi^{j}+\frac{\partial^{2} W}{\partial \xi^{i} \partial \xi^{j}} d \xi^{i} d \xi^{j}\right. \\
\left.+\left(\frac{\partial^{2} W}{\partial \chi^{i} \partial \xi^{j}}+\frac{\partial^{2} W}{\partial \chi^{j} \partial \xi^{i}}\right) d \chi^{i} d \xi^{j}\right) .
\end{array}
$$

In the case of an harmonic oscillator with frequency $\omega$, the functional $W$ reads

$$
W(\chi, \xi)=-\frac{1}{2}\left[\omega \chi^{2}+\omega^{-1} \xi^{2}\right] .
$$

The knowledge of the overlap suffices to construct the Hilbert space and the representation [29.

A vector of the Hilbert space can be constructed as a function on phase space of the form $\Psi(\chi, \xi)=\sum_{l} c_{l}\left\langle\chi \xi \mid \chi_{l} \xi_{l}\right\rangle$ for a finite number of complex numbers $c_{l}$ and $\chi_{l}, \xi_{l}$. The inner product between two vectors characterised by $c_{l}, \chi_{l}, \xi_{l}$ and $c_{l}^{\prime}, \chi_{l}^{\prime}, \xi_{l}^{\prime}$ is

$$
\sum_{l} c_{l}^{\prime *} c_{l}\left\langle\chi_{l}^{\prime} \xi_{l}^{\prime} \mid \chi_{l} \xi_{l}\right\rangle
$$

The Weyl group is then represented by the operators $\hat{U}(\chi, \xi)$, which are defined as

$$
\left(\hat{U}\left(\chi^{\prime}, \xi^{\prime}\right) \Psi\right)(\chi, \xi)=e^{\frac{i}{2}\left(\chi^{\prime} \cdot \xi-\xi^{\prime} \cdot \chi\right)} \Psi\left(\chi-\chi^{\prime}, \xi-\xi^{\prime}\right) .
$$

The above is written for the finite-dimensional Weyl group, but with little modification is also valid for the infinite dimensional case. The only difference is that in finite dimensions the Stone- von Neumann theorem holds: all irreducible, strongly continuous, unitary representations of the Weyl group, are unitarily equivalent.

In the infinite dimensional case the vector space out of which the Weyl group is constructed is a functional space. For field theories in Minkowski spacetime this is a subspace of the space of square integrable functions on $\mathbf{R}^{3}$. In this case, the group of spatial translations is also represented unitarily on the Hilbert space. If the vacuum is the unique translationary invariant state in the representing Hilbert space, then it can be proven that all unitarily equivalent representations share the same expectation functional, and conversely, if two representations differ in their expectation functionals, they are unitarily inequivalent [30]. We shall refer to this as the uniqueness theorem for the expectation functional.

\section{III.2 Classical histories}

In order to study the phase space structure of quantum mechanical histories, we need to describe histories in classical mechanics in a way that is amenable 
to a direct comparison. We shall, therefore, reproduce here the main points of this description, referring the reader to [11, 15] for details.

Consider the space of classical histories $\Pi$ viewed as the set of continuous paths on the classical phase space $\Gamma$. An element of $\Pi$ is a path $\gamma: T \rightarrow \Gamma$.

For any function $f$ on $\Gamma$ one can define a family of functions $F_{t}$ on $\Pi$ as

$$
F_{t}(\gamma)=f(\gamma(t)) .
$$

Taking for simplicity $\Gamma=\mathbf{R} \times \mathbf{R}=\{(q, p)\}$, we can define $q_{t}$ and $p_{t}$ as elements of $C^{\infty}(\Pi)$ through

$$
\begin{aligned}
& q_{t}(\gamma)=q(\gamma(t)), \\
& p_{t}(\gamma)=p(\gamma(t)) .
\end{aligned}
$$

Two other functions on $\Pi$ can be identified

$$
\begin{aligned}
V(\gamma) & =\int_{T} d t p_{t} \dot{q}_{t}(\gamma), \\
H(\gamma) & =\int_{T} d t h\left(p_{t}, q_{t}\right),
\end{aligned}
$$

with $h$ denoting the standard canonical Hamiltonian. If we furthermore equip $\Pi$ with a symplectic form

$$
\omega=\int d t d p_{t} \wedge d q_{t},
$$

corresponding to the Poisson bracket

$$
\left\{q_{t}, p_{t^{\prime}}\right\}=\delta\left(t, t^{\prime}\right),
$$

we can examine the canonical transformations generated by the functions $V$ and $H$. These are the generators of the two distinct laws of time transformation that characterize history theories.

The transformations generated by $V$ perform translations of the $t$ argument in a path, that is $\gamma \rightarrow \gamma^{\prime}$ with $\gamma^{\prime}(t)=\gamma(t+s)$ (s the affine parameter of the corresponding one-parameter group). Or in its induced action on the functions

$$
F_{t} \rightarrow F_{t+s} .
$$

$H$ respects the time labelling of the points of the path. It acts on each point of the path by transforming it (while keeping t fixed) according to Hamilton's equations. This means its action on $C^{\infty}(\Pi)$ is

$$
\left(q_{t}, p_{t}\right) \rightarrow\left(q_{t}(s), p_{t}(s)\right),
$$

where $q_{t}(s)$ is the function that to each path $\gamma$ assigns the $q$ - coordinate of the point obtained by integrating the Hamilton equations from initial point with coordinates $\left(q_{t}, p_{t}\right)$ to time $s$; similarly for $p_{t}(s)$. 
In the classical setting this distinction of two laws of time transformation, is nicely related to the least action principle. A path $\gamma$ is a solution to the classical equations of motions iff it is a fixed point of the canonical transformation generated by the action $S=V-H$. This implies the condition

$$
\left\{q_{t}, S\right\}(\gamma)=\left\{p_{t}, S\right\}(\gamma)=0 .
$$

Hence for the solutions to the equations of motion the laws of time evolution generated by $V$ and $H$ coincide.

\section{III.3 The history group}

The construction of the history Hilbert space through the tensor product of single-time Hilbert spaces suggests a natural generalisation; the history Hilbert space has to carry the representation of the history group, the history analogue of the canonical group [12]. This is a group that acts by symplectic transformations on the space of phase space histories. For linear phase spaces this is

$$
\left[q_{t}^{i}, p_{t^{\prime}}^{j}\right]=i \delta^{i j} \delta\left(t, t^{\prime}\right),
$$

It is clearly an infinite dimensional Weyl group. Its proper definition involves a choice of smearing functions: we define $q_{\xi}=\int d \mu(t) \xi_{i}(t) q_{t}^{i}$ and $p_{\chi}$ similarly, and write the commutator as

$$
\left[q_{\xi}, p_{\chi}\right]=i \int d \mu(t) \chi(t) \cdot \xi(t)
$$

The precise choice of a test-function space depends on the physics of the system, but it definitely has to consist of square-integrable functions, if the right-handside of (3.28) is to be defined. Here $d \mu$ stands for any measure on the real line, but what is mainly used is the measure employed in the construction of $\otimes_{t} H_{t}$, i.e. $d \mu(t)=d t / \tau$.

This history group is an infinite dimensional Weyl group and admits many unitarily inequivalent representations.

The analysis of the classical histories suggests the criterion for selecting a representations. There should exist self-adjoint operators in the Hilbert space, that correspond to the functions $V$ and $H$ of the classical theory. For quadratic Hamiltonians, a Fock representation (that has the structure of a continuous tensor product) can be constructed [13], in which both the Hamiltonian $H_{\kappa}$ and an operator corresponding to $V$ (the Liouville operator) exist. An important feature of this construction is the existence of a Hilbert space vector $|0\rangle$, which is the lowest eigenstate of the Hamiltonian and is left invariant under the action of $e^{i s V}[13,10]$. The projector $|0\rangle\langle 0|$ corresponds to the proposition that at all times the systems is to be found in the ground state.

Another important feature of this construction is the fact that the continuous tensor product of coherent states of the harmonic oscillator exists as a coherent state in the Fock Hilbert space. This is a feature that can be generalized for 
systems with non-quadratic Hamiltonian. Indeed, it will form the basis of our construction.

\section{III.3.1 General representations}

Representations cannot be explicitly constructed for non - quadratic Hamiltonians (it is the same situation with the one in canonical quantum field theory). Nonetheless, if we have some information about the canonical theory, we can exploit this to construct representations for the history group.

As we explained in section 2.3, unbounded operators can be defined on a history Hilbert space, if we start our construction from a subset $\mathcal{L}$ of the Hilbert space. Since we want a Hilbert space that carries a representation of the history group, the natural choice for $\mathcal{L}$ would be the coherent states of the corresponding canonical group. If $H$ carries a representation of the canonical group $\hat{U}(\chi, \xi)$ and $\hat{h}$ is the Hamiltonian with a unique ground state $|0\rangle_{H}$, we define the canonical coherent states $|z\rangle=|\chi \xi\rangle=\hat{U}(\chi, \xi)|0\rangle_{H}$. Then the history Hilbert space $\mathcal{V}$ is generated by all vectors

$$
|z(\cdot)\rangle=|\chi(\cdot) \xi(\cdot)\rangle:=\otimes_{t \in T}\left|\chi_{t} \xi_{t}\right\rangle_{H_{t}}
$$

Furthermore, we demand that the vectors $|z(\cdot)\rangle$ on $\mathcal{V}$ are the coherent states associated with the corresponding history group. In this case we shall have a history overlap kernel

$$
\left\langle\chi^{\prime}(\cdot) \xi^{\prime}(\cdot) \mid \chi(\cdot) \xi(\cdot)\right\rangle=\exp \left(\int d \mu(t) \log \left(\left\langle\chi_{t}^{\prime} \xi_{t}^{\prime} \mid \chi_{t} \xi_{t}\right\rangle_{H_{t}}\right)\right) .
$$

The corresponding expectation functional $K_{h}[\chi(\cdot), \xi(\cdot)]=e^{W_{h}[\chi(\cdot), \xi(\cdot)]}$ will read in terms of the canonical expectation functional $K(\chi, \xi)=e^{W[\chi, \xi]}$

$$
W_{h}[\chi(\cdot), \xi(\cdot)]=\int d \mu(t) W\left[\chi_{t}, \xi_{t}\right]
$$

Clearly certain conditions have to be imposed on the admissible paths $(\chi, \xi)(\cdot)$ if the integral is to be finite. (We shall take $T=\mathbf{R}$ without any loss of generality in this section.)

Now, there exists a norm $|\cdot|_{\Gamma}$ on the phase space (it can be constructed from the metric (3.7) or from the inner product). This induces a norm in the space of paths $t \rightarrow z_{t}$, which is given by

$$
|z(\cdot)|_{\Pi}=\int d \mu(t)\left|z_{t}\right|_{\Gamma} .
$$

Our first restriction, will be to consider only continuous paths with a finite value for the norm. For simplicity we shall assume that the maps $z(\cdot)$ take values $(0,0)$ except within compact subsets of $\mathbf{R}$. But we expect that our results would still be valid, if the paths $x(\cdot)$ converge to $(0,0)$ sufficiently fast (exponentially) outside compact sets. 
We shall also assume that the canonical coherent states, viewed as maps from the phase space to $H$ are smooth functions of their arguments. This implies that $W[\chi, \xi]$ is a smooth function of its variables. Since by definition $W[0,0]=0$, the above conditions are sufficient for the integral (3.31) to be finite.

We shall also impose the restriction that the maps $z(\cdot)$ are everywhere Lifschitz: in any compact subset $U$ of $\mathbf{R}$, there exists $C>0$, such that for all $t_{1}, t_{2} \in U,\left|z_{t_{1}}-z_{t_{2}}\right|_{\Gamma}<C\left|t_{1}-t_{2}\right|$. This is a stronger assumption than continuity, but weaker than differentiability and it is necessary for proving existence of the Liouville operator.

If $|\chi(\cdot) \xi(\cdot)\rangle$ are to correspond to coherent states, they have to be continuous functions of their arguments. This is proven as follows:

Let us assume that $\left|z_{1}(\cdot)-z_{2}(\cdot)\right|_{\Pi}=\delta>0$. Then

$$
\left.\| z_{1}(\cdot)\right\rangle-\left.\left|z_{2}(\cdot)\right\rangle\right|^{2}=2\left(1-\cosh \int d \mu(t) \log \left\langle z_{1 t} \mid z_{2 t}\right\rangle\right) .
$$

Let us write $\left|f_{t}\right\rangle=\left|z_{2 t}\right\rangle-\left|z_{1 t}\right\rangle$. Then we have

$$
||\left|z_{1}(\cdot)\right\rangle-\left|z_{2}(\cdot)\right\rangle \|_{\mathcal{V}}^{2}=2-2 \cosh \int d \mu(t) \log \left(1+\left\langle z_{t} \mid f_{t}\right\rangle\right) .
$$

The finiteness of $\left\|z_{1}(\cdot)-z_{2}(\cdot)\right\|_{\Pi}$, implies that except for a set of measure zero, there exists $c \geq 0$, such that $\left|\left\langle z_{t} \mid f_{t}\right\rangle\right| \leq \sqrt{\langle} f_{t}\left|f_{t}\right\rangle<c \delta$. Now, there exist complex numbers $c_{t}$, such that $\log \left(1+\left\langle z_{t} \mid f_{t}\right\rangle\right)=c_{t}\left\langle z_{t} \mid f_{t}\right\rangle$. By our previous result (except perhaps in a set of measure zero) these $c_{t}$ satisfy $\left|c_{t}\right|<C$, for $C>0$. Using this result, we get

$$
||\left|z_{1}(\cdot)\right\rangle-\left.\left|z_{2}(\cdot)\right\rangle\right|_{\mathcal{V}} ^{2}=2-2 \cosh \left(\int d \mu(t) c_{t}\left\langle z_{t} \mid f_{t}\right\rangle\right) .
$$

The integral is bounded $\left|\int d \mu(t) c_{t}\left\langle z_{t} \mid f_{t}\right\rangle\right| \leq C \delta$, so for sufficiently small $\delta$, there exists a constant $C^{\prime}>0$ such that

$$
\left|\| z_{1}(\cdot)\right\rangle-\left|z_{2}(\cdot)\right\rangle \|_{\mathcal{V}}^{2} \leq C^{\prime} \delta^{2}
$$

showing that $|z(\cdot)\rangle$ is a continuous function of $z(\cdot)$.

This implies that $W$ is also a continuous function of $\chi(\cdot), \xi(\cdot)$; so as explained in section 3.1.3, we define a representation of the history group using equation (3.16).

But the representation can also be defined straightforwardly. Indeed, we can write a unitary operator $U(\chi(\cdot), \xi(\cdot))$ as $\otimes_{t \in \mathbf{R}} U\left(\chi_{t}, \xi_{t}\right)$, i.e. by its action on the coherent state vectors

$$
U(\chi(\cdot), \xi(\cdot))\left|\chi^{\prime}(\cdot) \xi^{\prime}(\cdot)\right\rangle=e^{\frac{i}{2} \int d \mu(t)\left(\chi_{t}^{\prime} \cdot \xi_{t}-\chi_{t} \cdot \xi_{t}^{\prime}\right)}\left|\chi(\cdot)+\chi^{\prime}(\cdot), \xi(\cdot)+\xi^{\prime}(\cdot)\right\rangle .
$$

Therefore time averaged operators for position $q_{\xi}=\int d \mu(t) q_{t} \cdot \xi(t)$ and momentum $p_{\chi}=\int d \mu(t) p_{t} \chi(t)$ do exist on $\mathcal{V}$. 


\section{III.3.2 Operators}

Let us first see how we can define the analogue of the Hamiltonian $H_{\kappa}=$ $\int d \mu(t) h\left(q_{t}, p_{t}\right)$ in this Hilbert space.

Let $\hat{h}$ be the Hamiltonian on the canonical Hilbert space. We assume that the representation of the Weyl group can be chosen, so that all coherent state vectors lie in the domain of $\hat{h}$. This suffices to show that there exist complex numbers $A(s)$, such that

$$
\left\langle\chi^{\prime} \xi^{\prime}\left|e^{-i \hat{h} s}\right| \chi \xi\right\rangle=\left\langle\chi^{\prime} \xi^{\prime} \mid \chi \xi\right\rangle\left(1-i A(s) h\left(\chi, \xi ; \chi^{\prime}, \xi^{\prime}\right) s\right),
$$

where $h\left(\chi, \xi ; \chi^{\prime}, \xi^{\prime}\right)=\left\langle\chi^{\prime} \xi^{\prime}|\hat{h}| \chi \xi\right\rangle /\left\langle\chi^{\prime} \xi^{\prime} \mid \chi \xi\right\rangle$, and for each neighborhood of $s=0$ there exists $C>0$ such that $|A(s)| \leq C$. Let us try to define a version of the operator $U_{\kappa}(s)=e^{-i H_{\kappa} s}$ as $\otimes_{t} e^{-i \hat{h} \kappa(t) s}$. It is easy to show, as in section 2.3.3, that it is well-defined; the issue is to show it is continuous at $s=0$, for then by Stone's theorem $H_{\kappa}$ exists. We have

$$
\begin{array}{r}
\left|\left\langle\chi^{\prime}(\cdot) \xi^{\prime}(\cdot)|U(s)-1| \chi(\cdot) \xi(\cdot)\right\rangle\right| \\
=\left|\exp \left(\int d \mu(t) \log \left(\left\langle\chi_{t}^{\prime} \xi_{t}^{\prime}\left|e^{-i \hat{h} \kappa(t) s}\right| \chi_{t} \xi_{t}\right\rangle_{H_{t}}\right)\right)-1\right| \\
=\left|\exp \left(\int d \mu(t) \log \left(1-i A(s) s \kappa(t) h\left(\chi_{t}, \xi_{t} ; \chi_{t}^{\prime}, \xi_{t}^{\prime}\right)\right)\right)-1\right| \\
\leq C\left|\int d \mu(t) \kappa(t) h\left(\chi_{t}, \xi_{t}: \chi_{t}^{\prime}, \xi_{t}^{\prime}\right)\right||s| .
\end{array}
$$

Here $C$ is a real positive number. $U(s)$ has therefore matrix elements continuous with respect to $s$ if $\int d \mu(t) \kappa(t) h\left(\chi_{t}, \xi_{t} ; \chi_{t}^{\prime}, \xi_{t}^{\prime}\right)$ exists. We can take $\kappa(t)$ to be a measurable function that grows at most polynomially. If we have adjusted $\hat{h}$ so that $\hat{h}|0\rangle_{H}=0$, then it suffices that $h\left(\chi_{t}, \xi_{t} ; \chi_{t}^{\prime}, \xi_{t}^{\prime}\right)$ is continuous. For we have demanded that $\left(\chi_{t}, \xi_{t}\right) \rightarrow 0$ exponentially fast outside some compact set, hence $h\left(\chi_{t}, \xi_{t} ; \chi_{t}^{\prime}, \xi_{t}^{\prime}\right) \rightarrow 0$ outside this compact set.

The operator $H_{\kappa}$ can be therefore defined.

A Liouville operator corresponding to the classical function $\int d t p_{t} \dot{q}_{t}$ is also defined by its action on coherent states

$$
e^{i s V}|\chi(\cdot) \xi(\cdot)\rangle \rightarrow=\left|\chi^{\prime}(\cdot) \xi^{\prime}(\cdot)\right\rangle,
$$

where $\left(\chi^{\prime}(t), \xi^{\prime}(t)\right)=(\chi(t+s), \xi(t+s))$. We need to check that it is continuous at $s=0$. We have

$$
\left\langle\chi(\cdot) \xi(\cdot)\left|e^{i s V}\right| \chi(\cdot) \xi(\cdot)\right\rangle=\exp \left(\int d \mu(t) \log \left\langle\chi_{t} \xi_{t} \mid \chi_{t+s} \xi_{t+s}\right\rangle_{H_{t}}\right) .
$$

Since the coherent states are continuous functions of their arguments and the paths $(\chi, \xi)(\cdot)$ have been assumed Lifschitz, there exists a vector $\left|f_{t}, s\right\rangle_{H_{t}}$ on $H$ such that

$$
\left|\chi_{t+s} \xi_{t+s}\right\rangle_{H_{t}}=\left|\chi_{t} \xi_{t}\right\rangle_{H_{t}}+s\left|f_{t}, s\right\rangle_{H_{t}}
$$


and $\left\langle f_{t}, s \mid f_{t}, s\right\rangle<C_{t}$ for some constants $C_{t}>0$. Therefore

$$
\begin{array}{r}
\left|\left\langle\chi(\cdot) \xi(\cdot)\left|e^{i s V}-1\right| \chi(\cdot) \xi(\cdot)\right\rangle\right| \\
=\left|\exp \left(\int d \mu(t) \log \left(1+s\left\langle\chi_{t} \xi_{t} \mid f_{t}, s\right\rangle\right)_{H_{t}}\right)-1\right| \\
\leq A|s| \int d \mu(t) C_{t}
\end{array}
$$

for some constant $A>0$. Now, since we assume $\left(\chi_{t}, \xi_{t}\right) \rightarrow(0,0)$ outside compact intervals, $C_{t}$ can always be chosen to be constant in this compact interval and vanish outside this, thus rendering the integral finite. We therefore establish continuity of the matrix elements of $e^{i s V}$ around $s=0$.

The operator $V$, therefore, exists.

The existence of $V$ and $H_{\kappa}$ also implies the existence of an action operator $S_{\kappa}=V-H_{\kappa}$.

To summarize, assuming that:

1. the canonical coherent states are smooth functions of their arguments,

2. they lie in the domain of $\hat{h}$,

3. $\hat{h}$ has a unique ground state $|0\rangle_{H}$, in which $\hat{h}|0\rangle_{H}=0$

4. we consider paths $t \rightarrow z_{t}$, that satisfy the Lifschitz condition,

we can define a representation of the history group in a Hilbert space $\mathcal{V}$ in the fashion described, such that the two generators of time-transformation are self-adjoint operators on $\mathcal{V}$.

An issue that can be raised at this point is that the choice of paths is restricted to ones that $\left(\chi_{t}, \xi_{t}\right) \rightarrow 0$ as time goes to infinity. These are not sufficient to describe all conceivable phase space motions, as for instance this of oscillators that oscillate eternally. However, one can consider such properties in an arbitrarily large - but finite- time interval. This restriction is a consequence of the way we have chosen to define the smearing functions for the generators of the history group. A possibility that might lift this difficulty in a natural manner is briefly presented in section 4.1 .

\section{III.3.3 Uniqueness of the representation}

As $\left(\chi_{t}, \xi_{t}\right) \rightarrow(0,0)$ for large $t$, the only vector that is left invariant under the time translations generated by the Liouville operator is the "vacuum" vector $|0\rangle=\otimes_{t \in \mathbf{R}}|0\rangle_{H_{t}}$. (It corresponds to the proposition that the system is on the ground state at all times). Since the history Weyl group is isomorphic to the Weyl group of a field theory, we can use the uniqueness theorem for the expectation functional, to establish that any two of the representations, we have constructed are unitarily inequivalent, if they have different expectation functionals. 
This has different implications according to whether the canonical Weyl group is finite or infinite dimensional. If it is infinite dimensional and corresponds to a well behaved quantum field theory (i.e. with a unique translationally invariant vacuum), then the expectation functional of the canonical theory is independent of the representation and unique. Hence, the expectation functional for the history theory, constructed by equation (3.30) is also unique. This means for a given representation of the canonical group, we can obtain a representation of the history group, in such a way, that unitarily equivalent representations of the canonical group yield unitarily equivalent representations of the history group. This is, indeed, very satisfactory.

But for finite dimensional canonical Weyl group, all representations are unitarily equivalent. Hence different expectation functionals correspond to unitarily equivalent theories. But different expectation functionals canonically, lead to different expectation functionals for the history group. And these give rise to unitarily inequivalent representations. We are then in the unpleasant situation of having many inequivalent history theories corresponding to one canonical theory. There is no remedy for this. But, we should remark that the conditions developed throughout this section, constrain severely the choice of the representation of the canonical group, we are allowed to use. The canonical coherent states have to lie in the domain of all operators that we want to also define in the histories theory. Even if this does not guarantee uniqueness, at least it gives a guideline for which type of representations are interesting to use.

\section{III.3.4 The decoherence functional}

We saw that we have to restrict to paths $\left(\chi_{t}, \xi_{t}\right)$ that fall to zero rapidly at large $t$. This means that the single-time Hilbert space at $t= \pm \infty$ is essentially one dimensional, consisting only of the vector $|0\rangle$.

We saw that in the construction of the decoherence functional, the main problem came from the operators defined at the boundary Hilbert space. In this construction, when time is taken in the whole of the real line, the boundary Hilbert space is one-dimensional and the boundary operator $\mathcal{A}$ is just multiplicative. Hence the decoherence functional splits in the product of two phases:

$$
d(\alpha, \beta)=\operatorname{Tr}_{\mathcal{V}}\left(\mathcal{S U}^{\dagger} \alpha \mathcal{U}\right) \operatorname{Tr} \mathcal{V}\left(\mathcal{S}^{\dagger} \mathcal{U}^{\dagger} \beta \mathcal{U}\right)
$$

The operator $\mathcal{U}$ is easily identified as $e^{-i H_{\kappa}}$ for $\kappa(t)=t$.

The construction of the operator $\mathcal{S}$ is more intricate. Complex analyticity of the coherent states makes consideration of the diagonal matrix elements sufficient.

From the basic operation of the Weyl group we get that

$$
\left\langle\chi^{\prime} \xi^{\prime} \mid \chi \xi\right\rangle=\exp \left(i / 2\left(\xi \cdot \chi^{\prime}-\chi \cdot \xi^{\prime}\right)+W\left[\chi-\chi^{\prime}, \xi-\xi^{\prime}\right]\right)
$$

Assuming a discetization $t_{0}, t_{1}, \ldots t_{n}=t_{f}$ of the interval $\left[t_{i}, t_{f}\right]$ the definition (2.22) yields

$$
\left\langle\chi_{t_{0}}, \xi_{t_{0}} ; \ldots ; \chi_{t_{n}}, \xi_{t_{n}}|\mathcal{S}| \chi_{t_{0}}, \xi_{t_{0}} ; \ldots \chi_{t_{n}}, \xi_{t_{n}}\right\rangle=\left\langle\chi_{t_{0}} \xi_{t_{0}} \mid \chi_{t_{n}} \xi_{t_{n}}\right\rangle \prod_{i}\left\langle\chi_{t_{i}} \xi_{t_{i}} \mid \chi_{t_{i-1}} \xi_{t_{i-1}}\right\rangle
$$




$$
\begin{array}{r}
=e^{i / 2\left(\xi_{t_{n}} \cdot \chi_{t_{0}}-\chi_{t_{n}} \cdot \xi_{t_{0}}\right)+W\left[\chi_{t_{n}}-\chi_{t_{0}}, \xi_{t_{n}}-\xi_{t_{0}}\right]} \\
\times \exp \left(\sum_{i} \frac{i}{2}\left(\xi_{t_{i-1}} \cdot \chi_{t_{i}}-\xi_{t_{i}} \cdot \chi_{t_{i-1}}\right)+W\left[\chi_{t_{i-1}}-\chi_{t_{i}}, \xi_{t_{i-1}}-\xi_{t_{i}}\right]\right) \\
=e^{\frac{i}{2}\left(\xi_{t_{n}} \cdot \chi_{t_{0}}-\chi_{t_{n}} \cdot \xi_{t_{0}}\right)+W\left[\chi_{t_{n}}-\chi_{t_{0}}, \xi_{t_{n}}-\xi_{t_{0}}\right]} \\
\times \exp \left(\sum_{i} \frac{i}{2}\left[\xi_{t_{i}} \cdot\left(\chi_{t_{i}}-\chi_{t_{i-1}}\right)-\chi_{t_{i}} \cdot\left(\xi_{t_{i}}-\xi_{t_{i-1}}\right)\right]\right. \\
-\frac{\partial W}{\partial \xi}\left[\chi_{t_{i}}, \xi_{t_{i}}\right]\left(\xi_{t_{i}}-\xi_{t_{i-1}}\right)-\frac{\partial W}{\partial \chi}\left[\chi_{t_{i}}, \xi_{t_{i}}\right]\left(\chi_{t_{i}}-\chi_{t_{i-1}} \text { (III). } 46\right)
\end{array}
$$

Hence at the continuous limit get

$$
\begin{array}{r}
\langle\chi(\cdot) \xi(\cdot)|\mathcal{S}| \chi(\cdot) \xi(\cdot)\rangle=e^{\frac{i}{2}\left(\xi\left(t_{f}\right) \cdot \chi\left(t_{0}\right)-x\left(t_{f}\right) \cdot \xi\left(t_{0}\right)\right)+W\left[\chi\left(t_{f}\right)-\chi\left(t_{0}\right), \xi\left(t_{f}\right)-\xi\left(t_{0}\right)\right]} \\
\times \exp \left(\int_{t_{0}}^{t_{f}} d t \frac{i}{2}(\xi \cdot \dot{\chi}-\chi \cdot \dot{\xi})-\int_{W_{t_{0}}}^{W_{t_{f}}} d W\right)= \\
\exp \left(\frac{i}{2}\left(\xi\left(t_{f}\right) \cdot \chi\left(t_{0}\right)-\chi\left(t_{f}\right) \cdot \xi\left(t_{0}\right)\right)+W\left[\chi\left(t_{f}\right)-\chi\left(t_{0}\right), \xi\left(t_{f}\right)-\xi\left(t_{0}\right)\right]\right. \\
\left.-W\left[\chi\left(t_{f}\right), \xi\left(t_{f}\right)\right]+W\left[\chi\left(t_{0}\right), \xi\left(t_{0}\right)\right]\right) \\
\times \exp \left(\frac{i}{2} \int_{t_{0}}^{t_{f}} d t(\xi \cdot \dot{\chi}-\chi \cdot \dot{\xi})\right) \mathrm{I}
\end{array}
$$

Clearly as $\left[t_{0}, t_{f}\right] \rightarrow(-\infty, \infty)$ we get

$$
\langle\chi(\cdot) \xi(\cdot)|\mathcal{S}| \chi(\cdot) \xi(\cdot)\rangle=\exp \left(i \int_{-\infty}^{\infty} \xi \cdot \dot{\chi}\right)
$$

In particular, for a pair of coherent-state histories the decoherence functional reads

$$
d\left((\xi, \chi)(\cdot),\left(\xi^{\prime}, \chi^{\prime}\right)(\cdot)\right)=e^{i S[\xi(\cdot), \chi(\cdot)]-i S^{*}\left[\xi^{\prime}(\cdot), \chi^{\prime}(\cdot)\right]},
$$

where

$$
i S[\xi, \chi]=\left\langle\xi, \chi\left|\left(\frac{d}{d t}-i H\right)\right| \xi, \chi\right\rangle
$$

is the classical phase space action.

\section{III.4 The generating functional}

\section{III.4.1 N - point functions}

A probability theory does not only give probabilities to possible scenaria. It also provides expectation values for observables. In fact, a probability measure can be fully reconstructed from the knowledge of a sufficiently large number of expectation values: these are known as the moments of the distribution or in physics as the N-point functions. We shall write the relevant formulas in the context of stochastic processes, rather than single-time probability theory, for 
it is the analogue of these expressions that we shall attempt to generalize in the quantum context.

Let us denote by $x$ a vector that corresponds to a point of a sample space $\Omega$ and $\Omega^{T}$ the space of histories with elements the paths $x(\cdot)$. Let also $d \mu(x(\cdot))$ denote the probability measure in the space of paths. One then defines the $\mathrm{N}$ point functions

$$
G^{(n)}\left(a_{1}, t_{1} ; \ldots ; a_{n}, t_{n}\right)=\int d \mu(x(\cdot)) X_{t_{1}}^{a_{1}} \ldots X_{t_{n}}^{a_{n}}
$$

where $X_{t}^{a}(x(\cdot))=x^{a}(t)$ is a function on $\Omega^{T}$.

The information of the $\mathrm{N}$ - point functions is encoded in the generating functional

$$
Z[J]=\sum_{n=0}^{\infty} \frac{(i)^{n}}{n !} \int d t_{1} \ldots d t_{n} \sum_{a_{1} \ldots a_{n}} G^{(n)}\left(a_{1}, t_{1} ; \ldots ; a_{n}, t_{n}\right) J_{a_{1}}\left(t_{1}\right) \ldots J_{a_{n}}\left(t_{n}\right)
$$

The generating functional is just the Fourier transform of the stochastic measure

$$
Z[J(\cdot)]=\int d \mu(x(\cdot)) \exp \left(i \int d t X_{t}^{a} J_{a}(t)\right)
$$

The $N$-point functions (3.51) fully exhaust the physical content of the theory; hence the generating functional (3.53) provides a complete specification of the probability measure. In general, one can define generating functionals containing less complete information, e.g. ones that refer to one single observable. For instance given a function $f$ on $\Omega$ we can define

$$
Z_{f}[J(\cdot)]=\int d \mu(x(\cdot)) e^{i \int d t F_{t} J(t)}
$$

which generates the correlation functions of $f$. Or more generally, one can define generating functionals of time-averaged quantities $F$ (functions on $\Omega^{T}$ ) as

$$
Z_{F}(j)=\int d \mu(x(\cdot)) e^{i F(x(\cdot)) j},
$$

for some real number $j$.

\section{III.4.2 The CTP generating functional}

Since the decoherence functional is defined through bounded operators on $\mathcal{V}$, its definition can be extended to a bilinear functional over all bounded operators on $\mathcal{V}: d: B(\mathcal{V}) \times B(\mathcal{V}) \rightarrow \mathbf{C}$.

We shall first examine the discrete-time case. Let us consider an operator $\hat{A}$ on $H$. Then if $A_{t}$ denotes the corresponding single-time operator on $\mathcal{V}$ (see section 2.1), we can see that

$$
d\left(A_{t_{1}} \otimes A_{t_{2}}, 1\right)=\Theta\left(t_{1}-t_{2}\right) \operatorname{Tr}\left(\hat{\rho_{0}} \hat{A}\left(t_{1}\right) \hat{A}\left(t_{2}\right)\right)
$$




$$
\begin{array}{r}
+\Theta\left(t_{2}-t_{1}\right) \operatorname{Tr}\left(\hat{\rho}_{0} \hat{A}\left(t_{2}\right) \hat{A}\left(t_{1}\right)\right) \\
d\left(1, A_{t_{1}} \otimes A_{t_{2}}\right)=\Theta\left(t_{2}-t_{1}\right) \operatorname{Tr}\left(\hat{\rho} \hat{A}\left(t_{1}\right) \hat{A}\left(t_{2}\right)\right) \\
+\Theta\left(t_{1}-t_{2}\right) \operatorname{Tr}\left(\hat{\rho} \hat{A}\left(t_{2}\right) \hat{A}\left(t_{1}\right)\right)
\end{array}
$$

where $\hat{A}(t)$ is the Heisenberg picture operator on $H: e^{i \hat{H} t} \hat{A} e^{-i \hat{H} t}$. The right hand side of (3.56) and (3.57) are the time-ordered and anti-time-ordered twopoint function for this observable. Similarly we can construct higher timeordered and anti - time-ordered functions respectively, as well as mixed ones, e.g. $d\left(A_{t_{1}}, A_{t_{2}} \otimes A_{t_{3}}\right)$. They are usually denoted by $(r, s)$ correlation functions $r$ denoting the number of time-ordered and $s$ of anti-time-ordered appearances of $A$ in the expectation value. Such $N$ - point functions have been first used in the classic study of quantum Brownian motion by Schwinger [17]. They are obtained by an object known as the closed - time - path (CTP) generating functional [17, 31].

If we want to construct an object that encodes the information about the $N$-point functions at all times, we need to go to the continuum limit.

Let us by $A_{f}$ denote the time averaged version of an operator $\hat{A}$ on $H$, defined in the way we explained in section 2.3. Then we define the closed-timepath generating functional associated to the operator $\hat{A}$ as a function of a pair of smearing functions $J_{+}$and $J_{-}$through

$$
Z_{\hat{A}}\left[J_{+}(\cdot), J_{-}(\cdot)\right]=d\left(e^{i A_{J_{+}}}, e^{-i A_{J_{-}}}\right) .
$$

The signs + and - correspond respectively to the part that generates timeordered, vs anti-time-ordered correlation functions. In general the $(r, s)$ mixed correlation function for $A$ will be given by

$$
=\left.(-i)^{r} i^{s} \frac{\delta^{r}}{\delta J_{+}\left(t_{1}\right) \ldots \delta J_{+}\left(t_{r}\right)} \frac{\delta_{A}^{s}}{\delta J_{-}\left(t_{1}\right) \ldots \delta J_{-}\left(t_{s}\right)} Z\left[J_{+}, J_{-}\right]\right|_{J_{+}=J_{-}=0} .(1
$$

When the Hilbert space carries a representation $U(\chi, \xi)=e^{-i q_{\xi}-i p_{\chi}}$ of the history Weyl group, there exist time-averaged versions of the position and momentum operators. We can then construct the configuration space CTP generating functional as

$$
Z_{q}\left[\xi_{+}, \xi_{-}\right]=d\left(e^{i\left(q, \xi_{+}\right)}, e^{-i\left(q, \xi_{-}\right)}\right) .
$$

This generating functional has been widely used, mainly because it has a convenient path-integral expression. One can construct a corresponding effective action through a Legendre transform of $W=-i \log Z$ (known as the CTP effective action) [32].

But we can also write a generating functional that contains all phase space correlation functions. This is simply defined [14] as

$$
Z\left[\xi_{+}, \chi_{+} ; \xi_{-}, \chi_{-}\right]=d\left(U\left(\chi_{+}, \xi_{+}\right), U^{\dagger}\left(\chi_{-}, \xi_{-}\right)\right) .
$$


Since our representation of the history group is irreducible, all physical information about the physical system is contained in the CTP generating functional (3.61). Indeed, it is the quantum analogue of the generating functional (3.53) of a general stochastic process.

\section{III.5 The Wigner-Weyl transform}

\section{III.5.1 The canonical case}

In quantum mechanics a representation $\hat{U}(\chi, \xi)$ of the canonical group enables one to construct a linear map that takes a large class of Hilbert space operators to phase space functions. This is known as the Wigner-Weyl transform. It is implemented as follows: If $\hat{A}$ is a trace-class operator on $H$ then, we define the function $F_{\hat{A}}(q, p)$ on phase space as

$$
F_{\hat{A}}(q, p)=\int d \chi d \xi e^{-i \xi \cdot q-i \chi \cdot p} \operatorname{Tr}(\hat{U}(\xi, \chi) \hat{A}):=\operatorname{Tr}(\hat{\Delta}(q, p) \hat{A})
$$

where $\hat{\Delta}(q, p)=\int d \chi d \xi e^{-i \xi \cdot q-i \chi \cdot p} \hat{U}(\chi, \xi)$. This operator satisfies

$$
\int d q d p \hat{\Delta}(q, p)=\hat{1}
$$

and its matrix elements in a coherent state basis are given by

$$
\begin{aligned}
\left\langle\chi^{\prime} \xi^{\prime}|\Delta(q, p)| \chi \xi\right\rangle= & e^{i \xi \cdot\left(\chi-\chi^{\prime}\right)+i q \cdot\left(p-p^{\prime}\right)+i\left(\chi \cdot \xi-\chi^{\prime} \cdot \xi^{\prime}\right)} \\
& \times \tilde{K}\left[p+\frac{\xi+\xi^{\prime}}{2}, q-\frac{\chi+\chi^{\prime}}{2}\right],
\end{aligned}
$$

in terms of the Fourier transform of the expectation functional

$$
\tilde{K}[p, q]=\int d \mu(\chi, \xi) e^{-i \chi \cdot p-i \xi \cdot q} K[\chi, \xi] .
$$

Note that by $d q d p$ we denote the standard Lebesque measure on $\Gamma=\mathbf{R}^{2 n}$, normalized by a factor of $(2 \pi)^{-n}$.

This definition can be extended to bounded operators (at least when the Weyl group is finite dimensional ) and to a large class of unbounded ones. The Wigner-Weyl transform of a density matrix is known as the Wigner function. There are two important properties of the Wigner transform

$$
\begin{array}{r}
\int d q d p F_{\hat{A}}(q, p)=\operatorname{Tr}_{H} \hat{A} \\
\int d q d p F_{\hat{A}}(q, p) F_{\hat{B}}(q, p)=\operatorname{Tr}_{H}(\hat{A} \hat{B}) .
\end{array}
$$

The operator commutator induces on the phase the Moyal bracket $\{,\}_{M}$. For a pair of operators $\hat{A}$ and $\hat{B}$ their commutator $\hat{C}=[\hat{A}, \hat{B}]$ is associated with the symbol

$$
\frac{1}{i} F_{\hat{C}}=\left\{F_{\hat{A}}, F_{\hat{B}}\right\}_{M}:=2 F_{\hat{A}} \sin \left(\frac{1}{2}\{,\}\right) F_{\hat{B}}
$$


here $\{$,$\} is the Poisson bracket on phase space, written as a bilinear operator:$ $f\{\} g=,\{f, g\}$. The sinus in this expression refers to its Taylor series viewed as a function of this bilinear operator.

\section{III.5.2 The histories analogue}

We can proceed similarly in the histories case and to each trace-class operator $A$ on $\mathcal{V}$ associate a function $F_{A}$ on $\Pi$, the space of classical histories as

$$
F_{A}[\gamma]=F_{A}[q(\cdot), \xi(\cdot)]=\int D \xi(\cdot) D \chi(\cdot) e^{-i(q, \xi)(\gamma)-i(p, \chi)(\gamma)} \operatorname{Tr}(U(\xi, \chi) A) .
$$

This expression is only formal, since the measures $D \chi(\cdot)$ etc do not exist. What is implied is $F_{A}(\gamma)=\operatorname{Tr}_{\mathcal{V}}(A \Delta(q(\cdot), p(\cdot)))$. By $\Delta(q(\cdot), p(\cdot))$ we denote a linear map that is given by

$$
\Delta(q(\cdot), p(\cdot))=\otimes_{t} \hat{\Delta}\left(q_{t}, p_{t}\right) .
$$

If the operator $A$ is a product operator $\otimes_{t} \hat{A}_{t}$, then using equation (2.14) we see that

$$
F_{A_{f}}[q(\cdot), p(\cdot)]=\exp \left(\int d \mu(t) \log F_{\hat{A}_{t}}\left(q_{t}, p_{t}\right)\right)
$$

It is also easy to calculate the symbol for a time averaged operator $A_{f}$ by constructing the Weyl transform for $e^{i A_{f} s}$ and expanding around $s=0$. The result is

$$
F_{A_{f}}[q(\cdot), p(\cdot)]=\int d \mu(t) f(t) F_{\hat{A}}\left(q_{t}, p_{t}\right)
$$

Such is for instance the case of position, momentum operators and the Hamiltonian, so that

$$
\begin{aligned}
q_{f} & \rightarrow F_{q_{f}}=\int d \mu(t) q_{t} f(t), \\
p_{f} & \rightarrow F_{p_{f}}=\int d \mu(t) p_{t} f(t), \\
H_{\kappa} \rightarrow F_{H_{\kappa}} & =\int d \mu(t) \kappa(t) h\left(q_{t}, p_{t}\right),
\end{aligned}
$$

where $h(q, p)=F_{\hat{h}}(q, p)$ is the Wigner transform of the canonical Hamiltonian.

For more general operators on $\mathcal{V}$, the Weyl transform is effected by constructing first a suitable discrete-time expression in $\otimes_{i} H_{t_{i}}$ and then going to the continuum limit. It is more convenient to employ the decomposition of the unity for the canonical coherent states in order to compute the trace.

$$
\operatorname{Tr}_{\mathcal{V}} A=\int \prod_{i} d \mu\left(\chi_{t_{i}}, \xi_{t_{i}}\right)\left\langle\chi_{t_{1}} \xi_{t_{1}} ; \chi_{t_{2}} \xi_{t_{2}} \ldots \chi_{t_{n}} \xi_{t_{n}}|A| \chi_{t_{1}} \xi_{t_{1}} ; \chi_{t_{2}} \xi_{t_{2}} \ldots \chi_{t_{n}} \xi_{t_{n}}\right\rangle
$$


For operators that map coherent states into coherent states, the calculations are easier to perform. Such is, for instance, the operator $e^{i s V}$. We can compute

$$
e^{i s V} \rightarrow F_{e^{i s V}}=\int \prod_{t} d \mu\left(\chi_{t}, p_{t}\right)\left\langle\chi_{t} \xi_{t}\left|\hat{\Delta}\left(q_{t}, p_{t}\right)\right| \chi_{t+s} \xi_{t+s}\right\rangle_{H_{t}}
$$

If we expand this around $s=0$ we find that

$$
V \rightarrow F_{V}=\int d t p_{t} \dot{q}_{t}
$$

where the integral is of a Stieljes type.

Note that equations (3.69) and (3.76) are, as yet, defined for a discretization of the time interval. In order to compute any traces we will always need to check the finiteness of the expressions at the continuum limit. We will return to this later in the next subsection (3.5.3).

\section{III.5.3 The decoherence functional}

In an analogous manner, one can assign to the decoherence functional a "function" on $\Pi \times \Pi$ as

$$
\begin{array}{r}
W\left[q(\cdot), p(\cdot) \mid q^{\prime}(\cdot), p^{\prime}(\cdot)\right]=W\left[\gamma \mid \gamma^{\prime}\right]=\int D \xi_{+}(\cdot) D \chi_{+}(\cdot) D \xi_{-}(\cdot) D \chi_{-}(\cdot) \\
e^{-i\left(q, \xi_{+}\right)-i\left(p, \chi_{+}\right)+i\left(q^{\prime}, \xi_{-}\right)+i\left(p^{\prime}, \chi_{-}\right)} \times Z\left[\xi_{+}, \chi_{+} ; \xi_{-}, \chi_{-}\right]
\end{array}
$$

Given then some operators (these might be projectors that correspond to a history proposition) $A$ and $B$ on $\mathcal{V}$ we have

$$
d(A, B)=\int D \mu(\gamma) D \mu\left(\gamma^{\prime}\right) W\left[\gamma \mid \gamma^{\prime}\right] F_{A}(\gamma) F_{B}\left(\gamma^{\prime}\right)
$$

where $D \mu(\gamma)$ is a shorthand for $D \chi(\cdot) D \xi(\cdot)$.

In spite of the general non-definability of the integration measure, there is a very good sense in which the $W\left[\gamma \mid \gamma^{\prime}\right]$ exists : as the inductive limit of its discretetime expressions, in complete analogy with the Kolmogorov's construction of the stochastic probability measure. This proceeds as follows:

In standard quantum mechanics one can define objects that correspond to classical multi - time probabilities using the Wigner transform [33]. They are of the form

$W\left(q_{1}, p_{1}, t_{1} ; \ldots q_{n}, p_{n}, t_{n}\right)=\operatorname{Tr}\left(\hat{\rho}_{0} e^{i \hat{H} t_{1}} \hat{\Delta}\left(q_{1}, p_{1}\right) e^{-i \hat{H} t_{1}} \ldots e^{i \hat{H} t_{n}} \hat{\Delta}\left(q_{n}, p_{n}\right) e^{-i \hat{H} t_{n}}\right)$.

These distributions do not define a probability measure: they are complex and do not satisfy the Kolmogorov additivity condition. Rather they are the building blocks of the decoherence functional. In analogy with the stochastic case if we consider two discretizations $I=\left\{t_{1}, \ldots t_{n}\right\}$ and $I^{\prime}=\left\{t_{1}^{\prime} \ldots t_{m}^{\prime}\right\}$ of an interval $T$, we can define the objects the

$$
W_{n, m}\left[q_{1}, p_{1}, t_{1} ; \ldots q_{n}, p_{n}, t_{n} \mid q_{1}^{\prime}, p_{1}^{\prime}, t_{1}^{\prime} ; \ldots ; q_{m}^{\prime}, p_{m}^{\prime}, t_{m}^{\prime}\right]=\operatorname{Tr}\left(\hat{C}_{n}^{\dagger} \hat{\rho}_{0} \hat{C}_{m}^{\prime}\right) \text { (III }
$$


where

$$
\hat{C}_{m}=e^{i \hat{H} t_{1}} \hat{\Delta}\left(q_{1}, p_{1}\right) e^{-i \hat{H} t_{1}} \ldots e^{i \hat{H} t_{m}} \hat{\Delta}\left(q_{m}, p_{m}\right) e^{-i \hat{H} t_{m}}
$$

and similarly for $\hat{C}^{\prime}{ }_{m}$.

Let us as write $\Omega^{I}$ and $\Omega^{I^{\prime}}$ the spaces of discrete-time phase space histories. They can be equipped with the standard Lebesque measure $\prod_{t} d q_{t} d p_{t}$, so that $W_{n, m}$ can be used to define genuine decoherence functionals $d_{I, I^{\prime}}$ that satisfy properties (2.3). If we denote by $\Omega^{T}$ the space of phase space histories we can consider the injection map $i_{I, I^{\prime}}: \Omega^{n} \times \Omega^{m} \rightarrow \Omega^{T} \times \Omega^{T}$. These maps are measurable. It is easy to check that the hierarchy of functions $W_{n, m}$ satisfies an additivity condition

$$
\begin{array}{r}
\int d q_{t_{1}} d q_{t_{2}} W_{n, m}\left[q_{1}, p_{1}, t_{1} ; \ldots q_{n}, p_{n}, t_{n} \mid q_{1}^{\prime}, p_{1}^{\prime}, t_{1}^{\prime} ; \ldots ; q_{m}^{\prime}, p_{m}^{\prime}, t_{m}^{\prime}\right] \\
=W_{n-1, m}\left[q_{2}, p_{2}, t_{2} ; \ldots q_{n}, p_{n}, t_{n} \mid q_{1}^{\prime}, p_{1}^{\prime}, t_{1}^{\prime} ; \ldots ; q_{m}^{\prime}, p_{m}^{\prime}, t_{m}^{\prime}\right]
\end{array}
$$

In complete analogy to Kolmogorov's theorem, the above properties are sufficient to prove the existence of an additive, complex-valued, hermitian measure on $\Pi \times \Pi$, i.e. a decoherence functional $d_{\Pi}$, such that

$$
d_{I, I^{\prime}}=i_{I, I^{\prime}}^{*} d_{\Pi}
$$

It is important to remark that the definition of the decoherence functional on phase space took place with respect to the measurable subsets of $\Pi$, which define a Boolean algebra. This is clearly distinct from the logic of projectors on the Hilbert space $\mathcal{V}$. This is what enabled us to sidestep the non-definability of a decoherence functional from the discrete-time expressions.

This construction does not highlight the general structure of the decoherence functional. To see this, it is necessary to compute the Wigner transformations of the operators $\mathcal{S}$ and $\mathcal{U}$.

When the Hamiltonian is quadratic, the coherent states are Gaussians and the calculation of traces reduces to Gaussian integrals. In this case the functional relations of operators is preserved by the Weyl - Wigner transform. For the harmonic oscillator, we get

$$
\begin{aligned}
& \mathcal{U} \rightarrow F_{\mathcal{U}}=e^{-i H_{\kappa}}, \kappa(t)=t \\
& \mathcal{S} \rightarrow F_{\mathcal{S}}=\exp \left(-\frac{1}{2}\left[\omega\left(q_{t_{f}}-q_{t_{0}}\right)^{2}+\omega^{-1}\left(p_{t_{f}}-p_{t_{0}}\right)^{2}+i\left(p_{t_{f}} \cdot q_{t_{0}}-q_{t_{f}} \cdot p_{t_{0}}\right)\right]\right) \\
& \times e^{i / 2 \int_{t_{i}}^{t_{f}} d t\left(p_{t} \cdot \dot{q}_{t}-q_{t} \cdot \dot{p}_{t}\right)}
\end{aligned}
$$

In the case of more general Hamiltonians the calculations are more difficult to perform. But if we assume that the interval upon which histories are defined is the whole real line, the boundary condition forces that

$$
F_{\mathcal{S}}=e^{i \int d t p_{t} \dot{q}_{t}}
$$


The operator $\mathcal{U}$ is unitary, hence a transformation $A \rightarrow \mathcal{U} A \mathcal{U}^{\dagger}$ preserves the trace. The trace is also preserved by the Weyl - Wigner transform, hence on phase space $\mathcal{U}$ corresponds to a trace-preserving automorphism $\mathbf{T}$ of the algebra of functions on the space $\Pi$ of phase space paths. Explicitly this would be the continuum limit of

$$
\mathbf{T}=T_{t_{1}} \otimes \ldots \otimes T_{t_{n}}
$$

where $T_{t}$ corresponds to the automorphism of the algebra of single - time functions $A \rightarrow T_{t}[A]$ given by the Moyal bracket version of the Heisenberg equations of motion

$$
\frac{\partial}{\partial_{t}} T_{t}[A]=\left\{H, T_{t}[A]\right\}_{M}
$$

with $T_{0}[A]=A$.

In general the decoherence functional for phase space paths in a time interval $\left[t_{i}, t_{f}\right]$ will read

$$
d(A, B)=\int_{\Gamma_{t_{i}} \times \Gamma_{t_{f}}} d x \lambda_{x}^{*}\left(F_{A}\right) \lambda_{x}\left(F_{B}\right)
$$

where by

- $x$ we denote points on the boundaries $\Gamma_{t_{i}} \times \Gamma_{t_{f}}$. It is then a collective index for $\left(q_{t_{i}}, p_{t_{i}}, q_{t_{f}}, p_{t_{f}}\right)$. It is obtained by the Weyl - Wigner transform of the boundary operator with respect to the $r s$ indices in equation (2.19).

- $d x=d q_{t_{0}} d p_{t_{0}} d q_{t_{f}} d p_{t_{f}}$ is the standard measure on $\Gamma_{t_{0}} \times \Gamma_{t_{f}}$.

- $\lambda_{x}(\cdot)$ is a family of complex valued measures on the space of paths that have a functional dependence on boundary points $x$ which incorporates the actual initial state of the system. If by $\mathbf{T}$ we denote the automorphism generated by $\mathcal{U}$ then

$$
\lambda_{x}(A)=\int d \mu(\gamma) F_{\mathcal{A}^{x} \mathcal{S}}(\gamma) \mathbf{T}(A)(\gamma)
$$

In this equation $F_{\mathcal{A}^{x} \mathcal{S}}$ is the Weyl symbol associated with the operator $\mathcal{A}^{x} \mathcal{S}$ of equation (2.23). The colective variable $x$ again corresponds to the Weyl-Wigner transform of the indices $(r, s)$ of equation (2.19).

In this expression it is very clear that phases appear in the probability assignment solely because of the geometric phase encoded in the operator $\mathcal{S}$. This has been argued in [16], but in the present context it is clearer, since the automorphism $\mathbf{T}$ makes no reference to complex numbers in its definition. The presence of complex numbers in the decoherence functional is purely due to the presence of a $U(1)$ connection on phase space, as encoded in the function $F_{\mathcal{S}}$.

\section{III.6 The stochastic limit}

Rather than considering the decoherence condition (1.5) as a law of nature, that has to be exactly satisfied (as the consistent histories interpretation does), we can view it as a condition for the approximation of the physical system by a classical probabilistic theory. We remarked how the unequal time pseudo probability distributions $W_{n, m}$ do not satisfy the Kolmogorov additivity conditions. Perhaps a smeared version of them would (approximately) satisfy them 
so that one would get decoherence. So one can try to define smeared pseudo probability distributions like

$$
\begin{gathered}
\bar{W}_{n, 0}\left(\bar{q}_{1}, \bar{p}_{1}, t_{1} ; \ldots \bar{q}_{n}, \bar{p}_{n}, t_{n}\right) \\
=\int d q_{1} d p_{1} \ldots d q_{n} d p_{n} \chi_{\bar{q}_{1} \bar{p}_{1}}\left(q_{1}, p_{1}\right) \ldots \chi_{\bar{q}_{n} \bar{p}_{n}}\left(q_{n}, p_{n}\right) W_{n, 0}\left(q_{1}, p_{1}, t_{1} ; \ldots ; q_{n}, p_{n},\left(\mathrm{~A}_{n} I\right) .93\right)
\end{gathered}
$$

Here $\chi_{\bar{\chi}} \bar{\xi}$ denotes a smeared characteristic function of a cell centered around $\bar{\chi} \bar{\xi}$. This will depend on some parameters $V$ which will determine the volume of the cell, within which smearing is effected.

The objects $\bar{W}_{n, 0}$ are expectation values. They can be properly normalized if we divide them with the smearing volume. In that case they can be taken as the discrete-time probability densities that might correspond to a measure. If these smeared densities satisfy the Kolmogorov criterion, (which is to be expected in many systems given sufficient smearing) they would define an classical probability measure, that would give an effective stochastic description for the quantum system.

\section{III.6.1 General operators}

The above description is valid for general observables and not only the generators of the canonical group. Indeed if $\hat{A}$ is a self-adjoint operator with continuous spectrum $\Sigma$, one defines its corresponding generating functional $Z_{\hat{A}}\left[f_{+}, f_{-}\right]$as in equation (3.58). Now if $x \in \mathbf{R}$ denote points of the spectrum of $A$, we can construct a decoherence functional in the space of histories $x(\cdot): T \rightarrow \Sigma$ by an analogous expression to (3.78)

$$
W\left[x(\cdot) \mid x^{\prime}(\cdot)\right]=\int D f_{+}(\cdot) D f_{-}(\cdot) e^{-i\left(x, f_{+}\right)+i\left(x^{\prime}, f_{-}\right)} Z_{\hat{A}}\left[f_{+}, f_{-}\right]
$$

For any two functions $F$ and $G$ on the space of paths, we will have

$$
d(F, G)=\int D x(\cdot) D x^{\prime}(\cdot) F[x(\cdot)] G\left[x^{\prime}(\cdot)\right] W\left[x(\cdot) \mid x^{\prime}(\cdot)\right]
$$

The distribution $W$ can again be defined as the inductive limit of the discretetime distributions $W_{n, m}\left(x_{1}, t_{1} ; \ldots ; x_{n}, t_{n} \mid x_{1}^{\prime}, t_{1}^{\prime} ; \ldots ; x_{n}^{\prime}, t_{n}^{\prime}\right)$ as in equation (3.81), but with the operators

$$
\hat{\Delta}(x)=\int d J e^{-i x J} e^{i \hat{A} J}
$$

substituting $\hat{\Delta}(p, q)$.

Again one can look for the classical limit by constructing smeared characteristic functions $\chi_{\bar{x}}(x)$ for subsets of $\Sigma$. It is convenient to use a Gaussian function for $\chi_{\bar{x}}$. For instance

$$
\chi_{\bar{x}}(x)=\exp \left(-\frac{1}{2 \sqrt{V}}(x-\bar{x})^{2}\right)
$$




\section{III.6.2 Smearing}

Let us give a description of how the above prescription for finding the classical limit. We may start with discrete time histories with $n$ time -steps, which we shall simply call $t$ and consider the smearing functions $\chi_{\bar{x}}$.

$$
\chi_{\bar{x}(\cdot)}=\prod_{t} \chi_{\bar{x}_{t}}=\exp \left(-\frac{1}{2 \sqrt{V}} \sum_{t}\left(x_{t}-\bar{x}_{t}\right)^{2}\right)
$$

Then we evaluate the decoherence functional at a pair of $\chi$ 's (actually their corresponding positive operators) to be

$$
\begin{array}{r}
d\left(\chi_{\left.\bar{x}(\cdot), \chi_{\bar{x}^{\prime}(\cdot)}\right)=} \int D J_{+} D J_{-} Z_{\hat{A}}\left[J_{+}, J_{-}\right]\left(\int D x D x^{\prime} \chi_{\bar{x}(\cdot)}[x(\cdot)] \chi_{\bar{x}^{\prime}(\cdot)}\left[x^{\prime}(\cdot)\right] e^{-i\left(J_{+}, x\right)+i\left(J_{-}, x^{\prime}\right)}\right)\right. \\
=V^{n} \int D J_{+} D J_{-} Z_{\hat{A}}\left[J_{+} / \sqrt{V}, J_{-} / \sqrt{V}\right] \\
\times \exp \left(-\frac{1}{4}\left(J_{+}, J_{+}\right)-\frac{1}{4}\left(J_{-}, J_{-}\right)-i\left(\bar{x}, J_{+}\right)+i\left(\bar{x}^{\prime}, J_{-}\right)\right)
\end{array}
$$

By $\left(J, J^{\prime}\right)$ we imply here a discrete sum $\sum_{t} J_{t} J_{t}^{\prime}$. When, we go to the continuous limit it will imply $\int d \mu(t) J_{t} J_{t}^{\prime}$.

\section{III.6.3 The probability measure}

Assume now that with sufficient coarse - graining we can get approximate satisfaction of the decoherence condition for disjoint $\chi_{\bar{x}(\cdot)}$ and $\chi_{\bar{x}^{\prime}(\cdot)}$. The next step is to assume that the probabilities $p\left(\chi_{\bar{x}}\right)=d\left(\chi_{\bar{x}}, \chi_{\bar{x}}\right)$ can be used to define a probability measure

$$
p[\bar{x}(\cdot)]=\frac{1}{V^{n}} d\left(\chi_{\bar{x}}, \chi_{\bar{x}}\right)
$$

This is standard practice. It corresponds to the mathematical operation of extending a classical probability measure that is defined in only a part of the lattice of propositions (in this case a semi-lattice), to the whole of the lattice. This gives then a generating functional (note that $p[\bar{x}(\cdot)]$ has no multiplicative dependence on $V^{n}$ hence it is safe to go to the continuous limit)

$$
\begin{array}{r}
Z_{A}[J]=\int D \bar{x} p[\bar{x}(\cdot)] e^{i(\bar{x}, J)}= \\
\int D J_{+} \exp \left(-\frac{1}{2}\left(J_{+}, J_{+}\right)-\frac{1}{2}\left|J-J_{+}\right|^{2}\right) \\
\times Z\left[\left(J_{+}\right) / \sqrt{V},\left(J-J_{+}\right) / \sqrt{V}\right]
\end{array}
$$

This is the generating functional of a stochastic process for a classical observable $A$, that is obtained as the classical limit of a general quantum mechanical operator $\hat{A}$. 
The above construction can be repeated for phase space observables with no modifications. In this case, the representation of the canonical group, provides a natural metric on phase space, which can be used in order to construct smearing functions. In this case, a parameter analogous to $V$ plays the role of the volume of the phase space cell (with respect to this metric), within which one smears.

Details on how to obtain the classical stochastic limit of quantum systems with this method, together with a number of examples, are found in [34].

\section{III.7 Summary}

After giving a brief review of the canonical group construction and the histories version of classical mechanics, we showed how to construct a large class of representations of the history group, using coherent state techniques. A particular nice result was, that for well-behaved quantum field theories the representation of the canonical group uniquely determines one for the history group.

We then showed how to encode the correlation functions for generic observables of the theory into a CTP generating functional. The Wigner-Weyl transform offered a way of representing quantum mechanical objects on the phase space and define a continuous-time decoherence functional as the continuous limit of discrete-time ones.

Finally, we developed a general procedure for taking the classical probability limit of quantum mechanical histories.

\section{Discussion}

We shall now discuss a number of topics that explain or put into context the results of the previous two sections.

\section{IV.1 Time averaging}

First, we need to address a rather important issue, that we left uncommented. What is the role of the parameter $\tau$ that enters the definition of the time integral? It appeared there originally in order to render the measure dimensionless, so that operators $A_{f}$ would be dimensionally the same with their canonical counterparts $\hat{A}$.

In the case where $T$ is compact, we remarked that $\tau$ can be chosen as to normalize the measure to unity. But in the more general case, that $T=\mathbf{R}$, this cannot be done and one would have to accept $\tau$ as an additional parameter entering the histories quantum theory. On one hand, it would not appear into the physical predictions of the theory: the values of the decoherence functional are independent of $\tau$. Nonetheless, it would be present $\mathrm{n}$ the definition of the time averaged operators and perhaps in the physical correspondence with classical observables. 
One possible idea is to substitute all integrals over $d \mu(t)$ with the limit as $\tau \rightarrow \infty$ of $\frac{1}{\tau} \int_{-\tau / 2}^{\tau / 2} d t$. Classical quantities of the form

$$
q_{f}=\lim _{\tau \rightarrow \infty} \frac{1}{\tau} \int_{-\tau / 2}^{\tau / 2} d t q_{t} f(t)
$$

are more naturally interpreted as time-averaged values of the observable $q$. This implies that we can enlarge the space of possible test-functions. It would suffice to demand, that the smearing functions $f$ are constant outside a compact set (rather than zero), in order for the integral to be defined.

This could have as immediate consequence, that the boundary Hilbert spaces at infinities would not be one-dimensional, as is the case of when $f$ is of compact support.

But this would severely weaken our uniqueness theorem. We need to have a unique translationary invariant "vacuum" vector, in order for the uniqueness theorem to hold. This is not any more true, if $f$ is not a function of compact support: any vector $|z(\cdot)\rangle$ with constant values of $z(t)$ would be translationary invariant.

The representation theory for this history group would therefore be very different; in fact, the history group itself is different. Intuitively, one expects that the representation we would obtain from such a construction would be a reducible one: a direct integral of representations like the ones we constructed, each labelled by different boundary conditions for the coherent states as $t \rightarrow$ $\pm \infty$.

These considerations will be taken further in another paper.

\section{IV.2 The decoherence functional}

We tried various different ways to define a continuous-time decoherence functional. The straightforward analogy with Kolmogorov's construction failed, because we cannot continuously embed the lattice of single-time propositions to the lattice of history ones. We were then left with two choices: one is to incorporate the information about the initial condition in an object that is extended in time, rather than a density matrix as is the case in the canonical approach. This might be operationally meaningful (after all the initial state corresponds to a preparation that takes place in a time interval), but it contradicts our intuition that information about the system can be encoded at a single moment of time, without any need of knowing anything about its past history. (In a sense, such a construction might be considered as the violation of the analogue of the Markov condition for stochastic processes.)

The other alternative, is to define the decoherence functional with respect to the structure of propositions about phase space histories. This involves abandoning continuity, but in phase space the natural condition is measurability and using this we can construct a mathematically sensible continuous-time decoherence functional. Operationally it is a very satisfactory construction: phase space measurements exhaust the physical content of quantum theories. But 
one might raise the objection that we sacrificed the quantum logic structure of history propositions in order to achieve this.

This objection is valid, assuming one considers quantum logic to be a fundamental part of quantum theory. This is however an interpretational attitude towards quantum theory, which we do not feel obliged to adopt. But even should we concede this point, we could still argue, that the true quantum logic is the one corresponding to time-averaged history propositions, and the standard single-time one just an approximation.

It is nonetheless true, that our construction would be conceptually more complete, and aesthetically more satisfying, if we were able to provide a reconstruction theorem: that the knowledge of the decoherence functional on phase space, allows us to uniquely construct the Hilbert space of the theory, the decoherence functional defined as a bilinear functional on the histories Hilbert space and perhaps get some correspondence between phase space symmetries and quantum mechanical unitary operators. This would be an analogue of Wightman's reconstruction theorem in quantum field theory [35]: constructing the Hilbert space, the vacuum and the representations of the history group from the correlation functions. The analogy is very accurate, because the decoherence functional on phase space is equivalent to the CTP generating functional and thus incorporates information about all correlation functions.

So far, we have not been able to find a direct way to prove such a theorem. Of course, one could always proceed indirectly: define the Wightman functions from the CTP generating functional, from them the canonical Hilbert space, the vacuum and the Hamiltonian, and then repeat the construction of section 3 to construct the history Hilbert space and the representation of the history group. Even though this lends plausibility in the existence of a reconstruction theorem, it does not provide any physical or mathematical insight on the structure of history theories.

\section{IV.3 The classical limit}

The identification of the history Hilbert space was based on the representations of the history group. When we have a representation of a group, we inherit all structures associated to it: coherent states, their symbols and the Weyl Wigner transform. The phase space, then, appears as the most fundamental ingredient of the quantum theory.

Indeed, through the Weyl-Wigner transform we can cast quantum mechanical histories in a language that makes only indirect references to a Hilbert space and is completely based on classical phase space objects. This is important, because on phase space we know how to implement coarse-grainings, that are of interest for a wide class of physical systems.

For instance, in many particle systems, one could study coarse-grainings of the Boltzmann type (focusing on a description in terms of densities on a single-particle phase space) and derive their stochastic behavior, by the method described in 3.6. This might provide a way to proceed towards a declared aim of the consistent histories scheme: to find how hydrodynamic variables and their 
quasi-deterministic evolution laws arise from quantum theory [3, 36, 37, 38, 39]. In fact, all types of coarse-graining of classical statistical mechanics can be implemented for phase space histories.

Another area, where our results are relevant is in the study of back-reaction of quantum fields on geometry. The semiclassical treatment assumes that we can couple the Einstein tensor to the expectation value of a quantum stress-energy tensor.

For quantum fields in curved spacetime, the stress-energy tensor is not defined as an operator or even an operator-valued distribution on the Hilbert space of the theory. This is why it has to be renormalized [28], but, even so, one cannot remove the divergences from its correlation functions. Our construction suggests that one could first take the stochastic limit for the field in a histories version of the theory, and then construct a classical stress-energy tensor from the classicalized field. This would give a fully consistent scheme for dealing with the back-reaction of the matter to geometry, without the dangerous assumptions involved in computing expectation values of stress-energy tensors. This idea has been tentatively developed in [34].

\section{IV.4 Perturbation theory}

In practice, we cannot explicitly construct the Hilbert space of the theory and the basic objects for most interesting physical systems. That is, why we rely on approximation methods, like perturbation theory. In analogy to quantum field theory, we could perhaps develop a perturbation expansion for the decoherence functional, together with a renormalization scheme, in order to adequately treat non-linear systems

The first problem we would face, is the generic inadequacy of perturbation theory to deal with real-time evolution. In this case we have to expand the operator $e^{-i \hat{H} t}$ in powers of the coupling constant, something that becomes increasingly inaccurate with large values of $t$. In standard quantum theory, this problem is addressed by performing the perturbation expansion, not to the evolution operator, but to its resolvent $(E-\hat{H})^{-1}$, which is essentially its Fourier transform.

The CTP generating functional plays the same role, since it is a "Fourier transform" of the decoherence functional. In the CTP formalism, the perturbation theory is well defined -for instance, in the path integral representation and its accuracy does not depend on the time $t$. This leads to a perturbative evaluation of the decoherence functional, that does not suffer from the problems of real-time perturbation theory. As such, it provides a valuable tool for the construction of powerful approximation schemes in the histories programme.

\section{IV.5 The histories quantization programme}

The main motivation of this paper is to be found in the histories quantization programme. This aims to exploit the covariant nature and the richer content 
of the histories approach, in order to study quantum theories of systems with non-trivial temporal structure. The eventual aim is a theory of quantum gravity.

So far the programme has dealt with quantum fields in curved spacetime [14. 40] (where, unlike the canonical case, we can construct a theory accepting an instantaneous Hamiltonian) and with constrained systems. The two laws of time transformation have enabled a treatment of parameterized systems [15] (prototypes of general relativity), in which the problem of time does not appear. More recent results involve the more elaborate presence of Poincaré groups in quantum field theory [41 and the appearance of a representation of the spacetime diffeomorphism group in the histories version of general relativity [42].

The main obstacle to further generalisation has been the restriction to Fock representations for the history group, and hence only to quadratic systems. In this paper, we have constructed a larger class of representations and therefore enlarged the domain of applicability of the programme. We have also indicated, how a perturbative construction of history theories could be implemented.

This will provide tools for continuation of the programme: it will be possible to rigorously construct covariant quantum theories for a large class of systems, at least with the same level of rigor as the canonical approach.

\section{References}

[1] R. B. Griffiths. Consistent Histories and the Interpretation of Quantum Mechanics. J. Stat. Phys. 36: 219 (1984).

[2] R. Omnès. Logical Reformulation of Quantum Mechanics: I Foundations. J. Stat. Phys. 53: 893 (1988); The Interpretation of Quantum Mechanics. Princeton University Press, Princeton, 1994.

[3] M. Gell-Mann and J. B. Hartle. Quantum mechanics in the Light of Quantum Cosmology. In Complexity, Entropy and the Physics of Information, edited by W. Zurek. Addison Wesley, Reading, 1990; Classical Equations for Quantum Systems. Phys. Rev. D47: 3345, 1993.

[4] J. B. Hartle. Spacetime quantum mechanics and the quantum mechanics of spacetime. In Proceedings on the 1992 Les Houches School, Gravitation and Quantisation, 1993.

[5] F. Dowker and A. Kent. On the consistent histories approach to quantum mechanics. J. Stat. Phys. 82: 1575, 1996.

[6] A. Kent. Consistent sets yield contradictory inferences in quantum theory. Phys.Rev.Lett. 78: 2874, 1997.

[7] C. Anastopoulos. Quantum Theory without Hilbert Spaces. quantph/0008126, to appear in Found. Phys. 
[8] C.J. Isham. Quantum Logic and the Histories Approach to Quantum Theory. J. Math. Phys. 35:2157, 1994.

[9] C.J. Isham and N. Linden. Quantum Temporal Logic and Decoherence Functionals in the Histories Approach to Generalised Quantum Theory. J. Math. Phys. 35:5452, 1994.

[10] K. Savvidou. The Action Operator in Continuous Time Histories. J. Math. Phys. 40: 5657, 1999.

[11] K. Savvidou. Continuous Time in Consistent Histories. gr-qc/9912076.

[12] C.J.Isham and N. Linden. Continuous Histories and the History Group in Generalised Quantum Theory. J. Math. Phys. 36: 5392, 1995.

[13] C. Isham, N. Linden, K. Savvidou and S. Schreckenberg. Continuous Time and Consistent Histories. J. Math. Phys. 37:2261, 1998.

[14] C. Anastopoulos. Quantum Fields in Nonstatic Background: a Histories Perspective. J. Math. Phys. 41:617, 2000.

[15] K. Savvidou and C. Anastopoulos. Histories Quantisation of Parameterised Systems: I. Development of a General Algorithm. Class. Quant. Grav. $17: 2463,2000$.

[16] C. Anastopoulos and K. Savvidou. Quantum Mechanical Histories and the Berry Phase. quant-ph/0007093.

[17] J. S. Schwinger. Brownian Motion of a Quantum Oscillator. J. Math. Phys. 2, 407 (1961);

[18] C. J. Isham, N. Linden and S. Schreckenberg. The Classification of Decoherence Functionals: an Analogue of Gleason's Theorem. J. Math. Phys. 35: 6360, 1994.

[19] O. Rudolph and J.D.M. Wright. On Tracial Operator Representations of Quantum Decoherence Functionals. J. Math.Phys. 38: 5643, 1997.

[20] M. V. Berry. Quantal Phase Factors Accompanying Adiabatic Changes. Proc. Roy. Soc. Lond. A392:45, 1984.

[21] B. Simon. Holonomy, the Quantum Adiabatic Theorem and Berry's Phase. Phys. Rev. Lett. 51: 2167, 1983.

[22] D. Page. Phys. Rev. A 36: 3479, 1987.

[23] Y. Aharonov and J. Anandan. Phase Change During a Cyclic Quantum Evolution. Phys. Rev. Lett. 58: 1593, 1987.

[24] J. Samuel and R. Bhandari. General Setting for Berry Phase. Phys. Rev. Lett. 60: 2339, 1988. 
[25] S. Schreckenberg. Symmetry and History Quantum Theory: an Analogue of Wigner's Theorem. J. Math. Phys. 37: 6086, 1997. Symmetries of Decoherence Functionals, ibid 38: 759, 1997.

[26] C. J. Isham. Topological and Global Aspects of Quantum Theory. In Proceedings of the 1983 Les Houches School, Relativity, Groups and Topology II, 1983.

[27] F. A. Berezin. The Method of Second Quantization. Academic Press, New York, 1966.

[28] R. Wald. Quantum Field Theory in Curved Spacetime and Black Hole Thermodynamics. University of Chicago Press, Chicago, 1994.

[29] J. R. Klauder. A Coherent State Primer. in Coherent States: Applications in Physics and Mathematical Physics, edited by J. R. Klauder and B. Skagerstam. World Scientific, Singapore, 1985.

[30] H. Araki. Hamiltonian Formalism and the Canonical Commutation Relations in Quantum Field Theory. J. Math. Phys. 1: 492, 1960.

[31] L. V. Keldysh. Diagram Technique for Nonequilibrium Processes. Zh. Eksp. Teor. Fiz. 47: 1515, 1964.

[32] E. Calzetta and B. L. Hu. Closed Time Path Functional Formalism in Curved Spacetime: Application to Cosmological Backreaction Problems. Phys. Rev. D 35: 495, 1987.

[33] M.D. Srinivas. Quantum Mechanics as a Generalized Stochastic Process on Phase Space. Phys. Rev. D15: 2837, 1977.

[34] C. Anastopoulos. Quantum Correlation Function and the Classical Limit. gr-qc/0011111; to appear in Phys. Rev. D.

[35] R. F. Streater and A. S. Wightman. PCT, Spin, Statistics and All That. W. A. Benjamin, New York, 1964.

[36] T. A. Brun and J. J. Halliwell. Decoherence of Hydrodynamic Histories: A Simple Spin Model. Phys. rev. D54: 2899, 1996.

[37] J. J. Halliwell. Decoherent Histories and Hydrodynamic Equations. Phys. Rev. D58: 105015, 1998. Decoherent Histories and the Emergent Classicality of Local Densities. Phys. Rev. Lett. 83: 2481, 1999.

[38] T. A. Brun and J. B. Hartle. Classical Dynamics of the Quantum Harmonic Chain. Phys.Rev. D60: 123503, 1999.

[39] E. Calzetta and B. L. Hu. Influence Action and Decoherence of Hydrodynamic Modes. Phys. Rev. D59: 065018, 1999. 
[40] D. Noltingk. A Consistent Histories Approach to the Unruh Effect. grqc/0005063.

[41] N. Savvidou. Poincaré Invariance for Continuous-Time Histories. grqc/0104053.

[42] N. Savvidou. The Relation between the Spacetime Diffeomorphism Group and the Group of Constraints. (to appear) 